TRANSACTIONS OF THE

AMERICAN MATHEMATICAL SOCIETY

Volume 357, Number 11, Pages 4413-4443

S 0002-9947(05)03974-7

Article electronically published on June 9, 2005

\title{
FUNCTIONAL EQUATIONS AND THEIR RELATED OPERADS
}

\author{
VAHAGN MINASIAN
}

\begin{abstract}
Using functional equations, we define functors that generalize standard examples from calculus of one variable. Examples of such functors are discussed, and their Taylor towers are computed. We also show that these functors factor through objects enriched over the homology of little $n$-cubes operads and discuss the relationship between functors defined via functional equations and operads. In addition, we compute the differentials of the forgetful functor from the category of $n$-Poisson algebras in terms of the homology of configuration spaces.
\end{abstract}

\section{INTRODUCTION}

In the late eighties, Tom Goodwillie introduced in a series of papers (6], 7], [8]) a construction that plays the role of traditional Taylor series for functors of spaces. More precisely, for the given functor $F$, a tower of functors $\left\{P_{n} F\right\}$ was constructed with $P_{n} F$ of degree $n$. Moreover, this tower (when evaluated at a space $X$ satisfying certain connectivity conditions) approximates $F$ in some appropriate sense. Since then Goodwillie calculus has been used extensively, and new methods were developed to provide alternative descriptions in different categories. In particular, in [9], B. Johnson and R. McCarthy introduce a construction using cotriples that produces a Taylor tower of functors from any pointed category with coproducts to the category of chain complexes $C h(\mathbb{K})$ over a commutative ring $\mathbb{K}$.

Traditionally, after introducing the Taylor series for a function of one variable, one studies the Taylor series of simple functions, such as the exponential and logarithmic functions or the function $\frac{x}{1-x}$, since the derivatives of these functions can be easily computed. Not unexpectedly, computing Taylor towers of functors is more complicated. However some of the techniques from traditional real variable calculus apply. For example, if a one-variable function satisfies a sufficiently simple functional equation, its Taylor series can often be computed.

In [9], the authors observed that exponential functors are defined as a solution to a rather elementary functional equation, which allowed them to compute the cross effects and consequently, the layers (or fibers) of Taylor towers of these functors.

We follow this approach and define new classes of functors via functional equations. In addition to exponential functors, these are functors of type $\frac{x}{1-x}$, logarithmic functors and functors of type $f_{n}$, which mimic the one-variable functions $\frac{x}{1-x}$, $-\log (1-x)$, and $f_{n}=\left(1-\frac{x}{2^{n-1}}\right)^{-2^{n-1}}-1$, respectively.

Received by the editors June 16, 2003.

2000 Mathematics Subject Classification. Primary 55U15; Secondary 18D50, 55P99.

Key words and phrases. Little $n$-cubes operads, Goodwillie calculus, functional equations.

(C)2005 American Mathematical Society Reverts to public domain 28 years from publication 
A crucial observation is that forgetful functors from the category of (non-unital) commutative algebras, (non-unital) associative algebras, Lie algebras and $n$-Poisson algebras to chain complexes over $\mathbb{K}$ are examples of exponential functors, functors of type $\frac{x}{1-x}$, logarithmic functors and functors of type $f_{n}$, respectively. This leads to some interesting applications of our computations. For example, in [13] we follow the approach suggested in 9 and use the Taylor towers of exponential functors to derive the topological analogue (in the framework of [3]) of the commutative to associative spectral sequence, also known as the fundamental spectral sequence for THH.

In a joint work with R. McCarthy ([11]), functors of type $\frac{x}{1-x}$ are employed to develop a set up (analogous to that of Quillen in 14 for discrete case) which allows a discussion on homology and abelianization functors for $A_{\infty}$ algebras. Furthermore, specializing the results of ([11]) to functors of type $\frac{x}{1-x}$ or type $f_{n}$ and their towers gives us some splitting results for associative and $n$-Poisson algebras.

On a more curious point, this approach of associating algebraic structures with real variable functions gives an interesting angle of looking at some classical algebraic results. For example, the simple equation

$$
e^{-\log (1-x)}-1=\frac{x}{1-x}
$$

is at the root of the Poincaré-Birkhoff-Witt Theorem.

In other words, studying these functors of various types aids in understanding different algebraic structures, which suggests a relationship between such functors and some of the standard operads. More precisely, we construct a sequence of functors (defined as solutions to certain functional equations), namely functors of type $\frac{x}{1-x}, f_{2}, f_{3}, \cdots$, and exponential functors, which have the same set of algebras of different types associated with them as the homology of little $n$-cubes operads $\left\{e_{n}\right\}_{n=1}^{\infty}$, i.e. the associative algebras (for $e_{1}$ and functors of type $\frac{x}{1-x}$ ), $n$-Poisson algebras (for $e_{n}$ and type $f_{n}$ with $n \geq 2$ ) and commutative algebras (for $e_{\infty}$ and exponential functors).

In addition to understanding the functors of different types defined via functional equations, the exploration of this relationship with operads is the primary goal of this work.

The paper is structured as follows.

In Sections 1, 2, and 3 we set up the groundwork, as well as briefly recall some of the basic constructions from Goodwillie calculus, little $n$-cubes operads and the theory of cotriples, triples and algebras associated with them. References for indepth study of these objects are provided.

The rest of the paper is built with analytic functions of one variable in mind. In other words, the real variable function which is to be imitated is introduced. Then the corresponding functor is defined, followed by a discussion of the algebraic structures it carries and by examples. Thus, Section 4 focuses on exponential functors, Section 5 introduces functors of type $\frac{x}{1-x}$, and in Section 6 the Taylor towers of these functors are computed. In Section 7, we discuss logarithmic functors, as well as compute their Taylor towers. Section 8 deals with functors of type $f_{n}$, whose Taylor towers are computed in Section 9. Section 10 considers the set of different types as a whole. In addition, the relationship with the homology of little $n$-cubes operads is explored in this section. In particular, the forgetful functor 
from the category of $n$-Poisson algebras to chain complexes is considered in light of F. Cohen's computations of the homology of configuration spaces.

\section{Triples, cotriples and algebras}

Triples arise in category theory as an abstraction of the notion of an algebraic structure on an underlying space. Let $\mathcal{C}$ be a pointed category with coproducts.

Definition 1.1. A triple $(T, \mu, \eta)$ on $\mathcal{C}$ is a functor $T: \mathcal{C} \rightarrow \mathcal{C}$, together with natural transformations $\mu: T T \rightarrow T$ and $\eta: I d \rightarrow T$, such that the following diagrams commute:
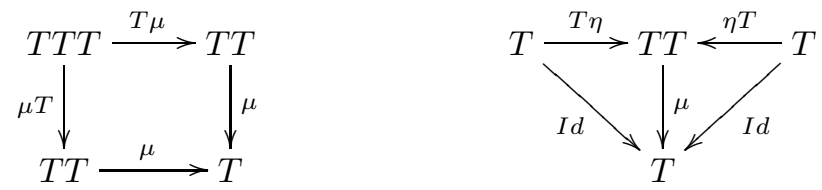

An algebra over a triple is a pair $(X, \rho)$, where $X$ is an object of the category $\mathcal{C}$ and $\rho: T X \rightarrow X$ is a morphism such that the composition $X \stackrel{\eta X}{\rightarrow} T X \stackrel{\rho}{\rightarrow} X$ is the identity and the obvious associativity diagram commutes. For more details we refer to Section 8.6 of [16].

Next we recall the definitions of a few common triples. For these examples, let $\mathcal{C}$ be a symmetric monoidal category, such as the category of modules over a commutative ring $\mathbb{K}$ or more generally, the category of chain complexes $C h(\mathbb{K})$. The latter category is where our subsequent work takes place. Consider the triple

$$
\mathbb{T}(X)=\bigoplus X^{\otimes n}
$$

In the category of modules, the algebras over this triple are precisely the associative algebras. Consequently, we will refer to algebras over this triple in any symmetric monoidal category as associative algebras. Similarly, the triple

$$
\mathbb{P}(X)=\bigoplus\left(X^{\otimes n}\right) / \Sigma_{n},
$$

where $\Sigma_{n}$ is the group of permutations, produces the commutative algebras. There are also triples associated to Lie algebras, $n$-Poisson algebras, and other structured objects.

There is a dual notion of cotriples $(\perp, \epsilon, \delta)$, which consist of a functor $\perp: \mathcal{C} \rightarrow \mathcal{C}$, together with a natural comultiplication map $\epsilon: \perp \rightarrow \perp \perp$ and a counit $\delta: \perp \rightarrow I d$, such that the obvious co-associativity and co-unit diagrams commute.

Adjoint pairs of functors $(F, U)$ provide a good source of cotriples, as the composite $F U$ is always a cotriple.

Remark 1.2. Let $(\perp, \epsilon, \delta)$ be a cotriple in $\mathcal{C}$. Fix an object $X$ in $\mathcal{C}$. Define $\perp^{*+1} X$ to be the simplicial object with $[n] \mapsto \perp^{n+1} X=\perp \cdots \perp X$, with face and degeneracy maps $s_{i}=\perp^{(i)} \delta \perp^{(n-i)}$ and $d_{i}=\perp^{(i)} \epsilon \perp^{(n-i)}$. We will recall this construction when presenting the definition of Goodwillie towers.

Chapter 8 of [16] is a good reference for an in-depth discussion of triples and cotriples. 


\section{LitTLE $n$-CUBES OPERADS}

Operads can be defined in any symmetric monoidal category. However, we will only consider the operads over $C h(\mathbb{K})$, the chain complexes over the commutative ring $\mathbb{K}$. An operad is a sequence of objects $\mathbf{a}(k), k \geq 0$, carrying an action of symmetric groups $\Sigma_{k}$, with products

$$
\mathbf{a}(k) \otimes \mathbf{a}\left(j_{1}\right) \otimes \cdots \otimes \mathbf{a}\left(j_{k}\right) \rightarrow \mathbf{a}\left(j_{1}+\cdots+j_{k}\right)
$$

which are equivariant and associative in an appropriate sense. For a precise definition along with a detailed discussion of operads we refer to [10] or [5].

Example 2.1. Fix a chain complex $V$. Define an operad with

$$
\mathcal{E}_{V}(k)=\operatorname{Hom}\left(V^{\otimes k}, V\right) .
$$

The symmetric group $\Sigma_{k}$ acts on $\mathcal{E}_{V}(k)$ through its action on $V^{\otimes k}$, and the structure maps are the obvious ones. More precisely, the morphism

$$
\mathcal{E}_{V}(k) \otimes \mathcal{E}_{V}\left(j_{1}\right) \otimes \cdots \otimes \mathcal{E}_{V}\left(j_{k}\right) \rightarrow \mathcal{E}_{V}\left(j_{1}+\cdots+j_{k}\right)
$$

maps the point $f \otimes f_{1} \otimes \cdots \otimes f_{k}$ (where $f: V^{\otimes k} \rightarrow V, f_{1}: V^{\otimes j_{1}} \rightarrow V, \cdots$, $\left.f_{k}: V^{\otimes j_{k}} \rightarrow V\right)$ to the function

$$
f \circ\left(f_{1} \otimes \cdots \otimes f_{k}\right): V^{\otimes j_{1}} \otimes \cdots \otimes V^{\otimes j_{k}} \rightarrow V^{\otimes k} \rightarrow V .
$$

Definition 2.2. An algebra over an operad a is a chain complex $A$ together with a morphism of operads $\rho: \mathbf{a} \rightarrow \mathcal{E}_{A}$. In other words, $A$ is equipped with structure maps

$$
\rho_{k}: \mathbf{a}(k) \otimes_{\Sigma_{k}} A^{\otimes k} \rightarrow A
$$

which are compatible in a natural sense. In other words, once again, the obvious associativity, unital and equivariance diagrams commute. See Chapter 2 of [10] for details.

Equivalently, an a-algebra can be defined as the algebra over the associated triple $T_{\mathbf{a}}(V)=\bigoplus \mathbf{a}(k) \otimes_{\Sigma_{k}} V^{\otimes k}$.

Next we briefly recall the definition and properties of some of the most important operads, the little n-cubes operads. For details, we again refer to [10] and [5].

Denote by $\mathbb{F}_{X}(S)$ the configuration space of embeddings of the finite set $S$ into the topological space $X$,

$$
\mathbb{F}_{X}(S)=\left\{x \in X^{S} \mid x(l) \neq x(t), l \neq t\right\} .
$$

Abbreviate $\mathbb{F}_{\mathbb{R}^{n}}$ by $\mathbb{F}_{n}$. Let $s$ be the number of elements of $S$, and define $\mathbf{e}_{n}(s)$ to be the homology of the configuration space $H\left(\mathbb{F}_{n}(S), \mathbb{K}\right)$, for $s>0$, and $\mathbf{e}_{n}(0)=0$. $\mathbf{e}_{n}(s)$ has an obvious action of $\Sigma_{s}$, and the sequence $\left\{\mathbf{e}_{n}(s)\right\}$ assembles into an operad. We call an algebra for the operad $\mathbf{e}_{n}(s)$ an $n$-algebra and denote the associated triple by $T_{n}$.

Observe that the configuration space $\mathbb{F}_{1}(k)$ is a disjoint union of contractible spaces; the components correspond to permutations $\sigma \in \Sigma_{k}$ by the rule

$$
\sigma \longmapsto\left\{\left(x_{\sigma(1)}, \cdots, x_{\sigma(k)}\right) \in \mathbb{R}^{k} \mid x_{\sigma(1)}<\cdots<x_{\sigma(k)}\right\} .
$$

It follows that $\mathbf{e}_{1}(k)=\mathbb{K}\left[\Sigma_{k}\right]$, and the associated triple is the associative algebra triple $T_{1}(V)=\bigoplus V^{\otimes k}$.

The configuration spaces of $\mathbb{R}^{\infty}$ are contractible. Hence the triple associated to $\mathbf{e}_{\infty}$ is the symmetric algebra triple $T_{\infty}(V)=\bigoplus\left(V^{\otimes k}\right)_{\Sigma_{k}}$. 


\section{Goodwillie calculus}

In this section we give a brief summary of the main constructions of Goodwillie calculus as developed by B. Johnson and R. McCarthy. For details we refer to $[9$, which is an application of [6], 7] and [8].

We begin by recalling the definition of the cross effects of a functor $F: \mathcal{C} \rightarrow \mathcal{A}$, where $\mathcal{C}$ is a pointed category with finite coproducts and $\mathcal{A}$ is an Abelian category.

Definition 3.1. The $n$ 'th cross effect of $F$ is the functor $c r_{n} F: \mathcal{C}^{\times n} \rightarrow \mathcal{A}$ defined inductively by

$$
c r_{1} F(M) \oplus F(*) \cong F(M),
$$

where $*$ is the initial/final object of the category.

$$
c r_{2} F\left(M_{1}, M_{2}\right) \oplus c r_{1} F\left(M_{1}\right) \oplus c r_{1} F\left(M_{2}\right) \cong c r_{1} F\left(M_{1} \vee M_{2}\right)
$$

and, in general,

$$
c r_{n} F\left(M_{1}, \cdots M_{n}\right) \oplus c r_{n-1} F\left(M_{1}, M_{3} \cdots M_{n}\right) \oplus c r_{n-1} F\left(M_{2}, M_{3} \cdots M_{n}\right)
$$

is equivalent to

$$
c r_{n-1} F\left(M_{1} \vee M_{2}, M_{3} \cdots M_{n}\right) .
$$

Definition 3.2. Given a functor $F$ from $\mathcal{C}$ to $C h(\mathcal{A})$, we say that $F$ is degree $n$ if $c r_{n+1} F$ is acyclic as a functor from $\mathcal{C}^{\times n+1}$ to $C h(\mathcal{A})$. That is, $c r_{n+1} F$ has no homology when evaluated on a collection of $n+1$ objects in $\mathcal{C}$.

In particular we say that $F$ is linear if it is of degree one.

Recall that functor $F: \mathcal{B} \rightarrow \mathcal{A}$ of pointed categories is reduced if it maps the initial/final object of $\mathcal{B}$ to the initial/final object of $\mathcal{A}$.

If in addition the source category $\mathcal{C}$ happens to be the symmetric monoidal category $C h(\mathcal{A})$, i.e. $F$ is an endofunctor, then in order for $F$ to be reduced and linear, in other words to preserve the initial object and coproducts, it has to be equal to the functor $-\otimes X$ for some $X \in C h(\mathcal{A})$. For reference purposes, we list this simple result in a form convenient to us in the following lemma.

Lemma 3.3. Let $F$ be a reduced linear endofunctor of $C h(\mathcal{A})$. Then for all $X \in$ $C h(\mathcal{A})$

$$
F(X) \cong F(X \otimes \mathbb{K}) \cong X \otimes F(\mathbb{K})
$$

where $\mathbb{K}$ is the monoidal unit of $C h(\mathcal{A})$.

Denote the category of functors of $n+1$ variables from $\mathcal{C}$ to $\mathcal{A}$ that are reduced in each variable by $\operatorname{Func}_{*}\left(\mathcal{C}^{\times n+1}, \mathcal{A}\right)$. Let $\triangle^{*}$ be the functor from $F u n c_{*}\left(\mathcal{C}^{\times n+1}, \mathcal{A}\right)$ to $F \operatorname{unc}_{*}(\mathcal{C}, \mathcal{A})$ obtained by composing a functor with the diagonal functor from $\mathcal{C}$ to $\mathcal{C}^{\times n+1}$. The $(n+1)$ st cross effect is the right adjoint to $\triangle^{*}$.

Definition 3.4. Let $\perp_{n+1}=\triangle^{*} \circ c r_{n+1}$ be the cotriple on $F u n c_{*}(\mathcal{C}, \mathcal{A})$ obtained from the adjoint pair $\left(\triangle^{*}, c r_{n+1}\right)$. We define $P_{n}$ to be the functor from $F u n c_{*}(\mathcal{C}, \mathcal{A})$ to $\operatorname{Func}_{*}\left(\mathcal{C}, C h_{\geq 0} \mathcal{A}\right)$ given by

$$
P_{n} F=\text { hocofiber }\left[N\left(\perp_{n+1}^{*+1}\right) \stackrel{\epsilon}{\rightarrow} i d\right],
$$

where $N$ is the associated normalized chain complex of the simplicial object, and $\epsilon$ is the map derived from the adjunction unit. Furthermore, let $p_{n}: i d \rightarrow P_{n}$ be the natural transformation obtained from the mapping cone. 
Next we produce a natural transformation $q_{n}: P_{n} \rightarrow P_{n-1}$.

Observe that we have the following formula relating the $n$ 'th and $n+1$ 'st cross effects:

$$
c r_{n+1} F\left(X_{1}, \cdots, X_{n+1}\right)=c r_{2}\left(c r_{n} F\left(X_{1}, \cdots, X_{n-1},-\right)\right)\left(X_{n}, X_{n+1}\right) .
$$

Fix an object $X$ of $\mathcal{C}$. Let $G(Y)=c r_{n} F(X, \cdots, X, Y)$. Then using the fold map, we have $\mathrm{cr}_{2} G(X, X) \rightarrow G(X \vee X) \rightarrow G(X)$, which in turn gives us

$$
c r_{2}\left(c r_{n} F(X, \cdots, X,-)\right)(X, X) \rightarrow c r_{n} F(X, \cdots, X, X) .
$$

Combine this map with the Equation (1) to produce a map $c r_{n+1} F(X, \cdots, X) \rightarrow$ $c r_{n} F(X, \cdots, X)$, which induces the desired map $q_{n}$. By Lemma 2.11 and Theorem 2.12 of $[9]$ these assemble into a tower

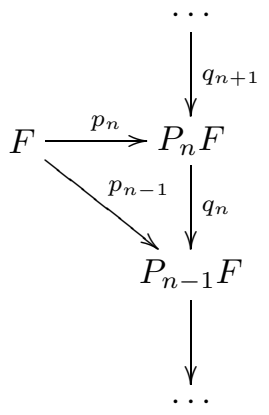

such that

1) The functor $P_{n} F$ is degree $n$.

2) If $F$ is degree $n$, then $p_{n}: F \rightarrow P_{n} F$ is a quasi-isomorphism.

3) The pair $\left(P_{n}, p_{n}\right)$ is universal up to natural quasi-isomorphism with respect to degree $n$ functors with natural transformations from $F$.

Definition 3.5. The $n$ 'th layer or the $n$ 'th derivative of $F$ is the functor

$$
D_{n} F(-)(X):=\text { hofiber }\left(q_{n}\right)(X) \text {. }
$$

It is worth noting that the above definition is a slight digression from the terminology typically used in Calculus of Functors, as the word 'derivative' is usually reserved for the classifying spectrum of $D_{n} F$ as opposed to the functor $D_{n} F$ itself (e.g. see $[8]$ ).

\section{Exponential FUnCTORS}

In this section we define the first class of functors that we are interested in (exponential functors), and discuss a few of their properties. The definition of exponential functors goes back to S. Mac Lane. However we have learned it from [9], where the authors also prove the following structure lemma. Here we present a (different) proof, which is better aligned with the rest of this work.

Reduced functions of real variables (i.e. functions $f(x)$ with $f(0)=0$ ) will serve as a motivation for our definitions. Observe that functions of the form $f(x)=$ $\exp (a x)-1$ satisfy the functional equation

$$
f(x) f(y)+f(x)+f(y)=f(x+y) .
$$


Moreover, every reduced analytic function that satisfies the above equation is of the form

$$
f(x)=\exp (a x)-1=(\exp (x)-1) \circ(a x) .
$$

Thus, we give the following definition.

Definition 4.1. A reduced functor $F$ from $\mathcal{C}$ to $C h(\mathbb{K})$ is exponential if there is a natural isomorphism

$$
\alpha_{X, Y}: F(X) \otimes F(Y) \oplus F(X) \oplus F(Y) \rightarrow F(X \vee Y)
$$

such that for all objects $X$ and $Y$ of $\mathcal{C}$ the following diagram naturally commutes:

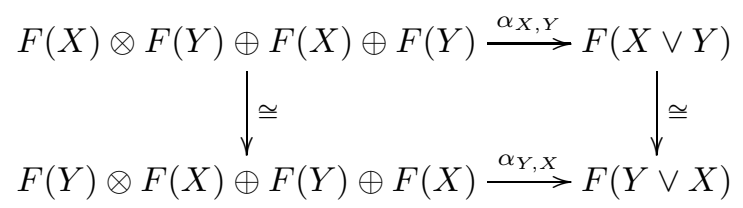

Example 4.2. Let $U_{e}$ be the forgetful functor from the category $\mathcal{N}_{\mathbb{K}}$ of non-unital commutative differential graded $\mathbb{K}$-algebras to the category $C h(\mathbb{K})$ of chain complexes. Observe that the coproduct of objects $X$ and $Y$ in $\mathcal{N}_{\mathbb{K}}$ is given by

$$
X \otimes Y \oplus X \oplus Y .
$$

Thus the functor $U_{e}$ is exponential.

In a certain sense $U_{e}$ is the only example of exponential functors. More precisely, continuing with the analogy with functions of real variables, we prove the following lemma.

Lemma 4.3. Every exponential functor $F$ from $\mathcal{C}$ to $C h(\mathbb{K})$ can be factored as $F=U_{e} \circ \tilde{F}$, where $\tilde{F}$ is a reduced coproduct-preserving functor from $\mathcal{C}$ to $\mathcal{N}_{\mathbb{K}}$.

Note that this lemma is the analogue of the earlier statement that every reduced analytic function satisfying the exponential equation is of the form $(\exp (x)-1) \circ$ $(a x)$, as the role of $\exp (x)-1$ is played by $U_{e}$, and the reduced coproduct-preserving functor $\tilde{F}$ is the appropriate analogue of the term $a x$.

Proof. Observe that the statement that $F$ can be factored as $F=U_{e} \circ \tilde{F}$ is equivalent to the claim that $F(X)$ is equipped with a natural structure of a non-unital commutative algebra. Thus our strategy will be to produce a natural multiplication map on $F(X)$ which is both commutative and associative.

Define a product $\mu: F(X) \otimes F(X) \rightarrow F(X)$ by the composite

$$
F(X) \otimes F(X) \longrightarrow F(X) \otimes F(X) \oplus F(X) \oplus F(X) \stackrel{\alpha_{X, X}}{\longrightarrow} F(X \vee X) \stackrel{F(+)}{\longrightarrow} F(X)
$$

As $\mu$ is commutative (obvious from the definition of an exponential functor), we only need to show that that it is associative. By naturality, we have a pair of commutative diagrams

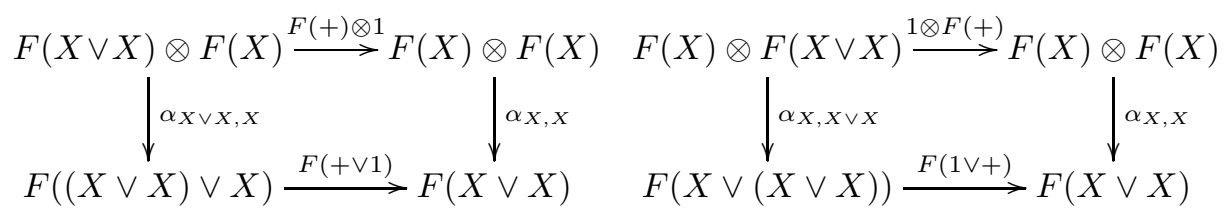


Thus, it is sufficient to prove that the diagram

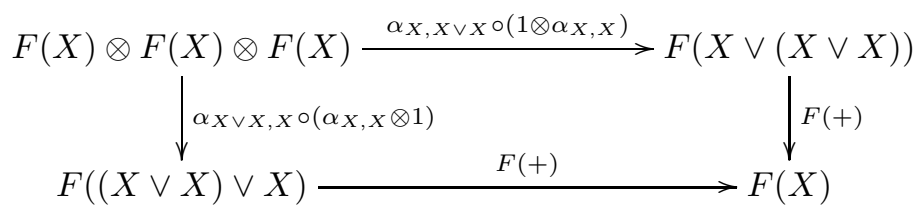

commutes.

To show that Diagram (2) commutes, we produce a diagonal map

$$
p: F((X \vee X) \vee X) \rightarrow F(X \vee(X \vee X))
$$

(induced by a permutation on $X \vee X \vee X$ ) that makes both the top left and bottom right triangles of Diagram (2) commute.

The following series of equivalences, which follow from the defining equation of exponential functors, provides a description of the top horizontal arrow in Diagram (2):

$$
\begin{aligned}
F(X) & \otimes F(X) \otimes F(X) \oplus 3 F(X \vee X) \\
1 \oplus 2 \alpha_{X, X}^{-1} \oplus 1 & \cong F(X) \otimes F(X) \otimes F(X) \oplus 2 F(X) \otimes F(X) \oplus 4 F(X) \oplus F(X \vee X) \\
& \cong F(X) \otimes[F(X) \otimes F(X) \oplus F(X) \oplus F(X)] \oplus 4 F(X) \oplus F(X \vee X) \\
1_{F(X)} \otimes \alpha_{X, X} \oplus 1 & \cong F(X) \otimes F(X \vee X) \oplus 4 F(X) \oplus F(X \vee X) \\
& \cong F(X \vee X \vee X) \oplus 3 F(X) .
\end{aligned}
$$

This and an analogous series of equivalences which describe the left vertical map of Diagram (21) are employed to form the following diagram:

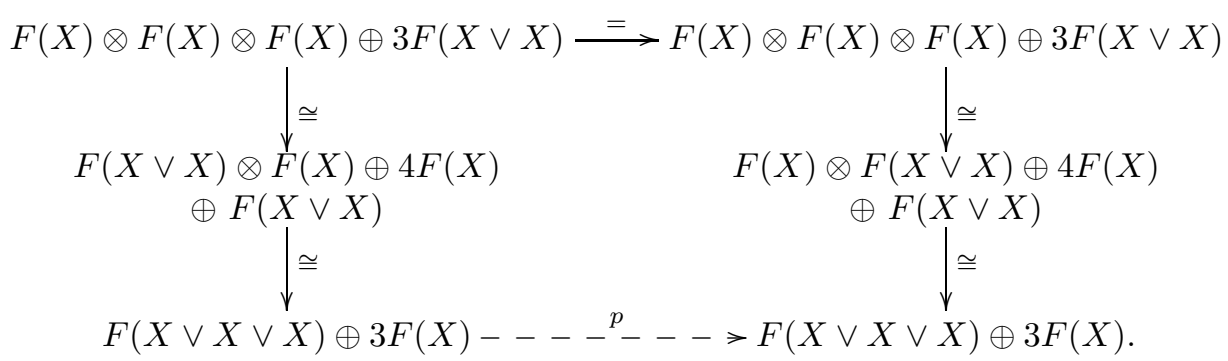

The dotted arrow $p$ exists simply because all the other maps in the diagram are isomorphisms, and by description of the two vertical maps provided above, it is just the functor $F$ applied to a permutation of $X \vee X \vee X$. Of course, the maps $X \vee X \vee X \stackrel{\rightarrow}{\rightarrow} X$ and $X \vee X \vee X \stackrel{\text { perm }}{\rightarrow} X \vee X \vee X \stackrel{+}{\rightarrow} X$ (where perm is some permutation) are the same, and observing that the two maps are still equal after 
we apply $F$ to the maps (as $F$ is a functor), we have a commutative diagram:

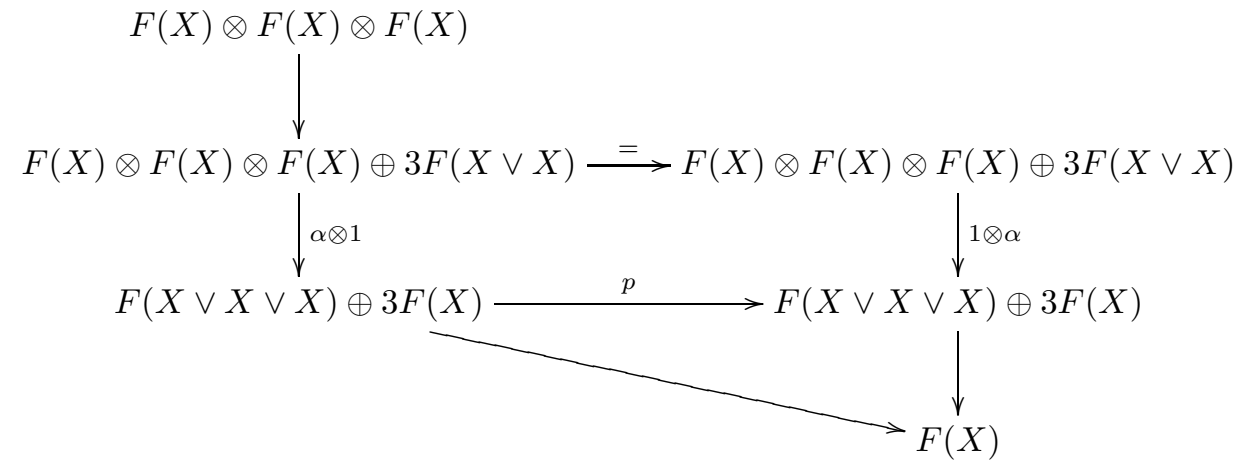

which shows that the Diagram (2) commutes and completes the proof of associativity of $\mu$.

It remains to prove that the functor $\tilde{F}$ from $\mathcal{C}$ to $\mathcal{N}_{\mathbb{K}}$ that we just obtained is coproduct preserving. Observe that it is immediate from the universal property of coproducts that $F(X) \otimes F(Y) \oplus F(X) \oplus F(Y)$ is the coproduct of $F(X)$ and $F(Y)$ in $\mathcal{N}_{\mathbb{K}}$ and hence $\tilde{F}$ is coproduct preserving.

\section{FUnCtORS OF TYPE $\frac{x}{1-x}$}

The next class of functors we consider mimics functions $f(x)=\frac{1}{1-a x}-1$. Here, as before, we subtract 1 to make the functions reduced. These functions are determined by the functional equation

$$
f(x)+f(y)+2 f(x) f(y)+f(x+y) f(x) f(y)=f(x+y) .
$$

Moreover, every reduced analytic function that satisfies the above equation is of the form $f(x)=\left(\frac{1}{1-x}-1\right) \circ(a x)$. This leads us to the following definition.

Definition 5.1. A reduced functor $F$ from $\mathcal{C}$ to $C h(\mathbb{K})$ is of type $\frac{x}{1-x}$ if there is a natural isomorphism

$\alpha_{X, Y}: F(X) \oplus F(Y) \oplus$

$$
[F(X) \otimes F(Y)] \oplus[F(Y) \otimes F(X)] \oplus[F(X \vee Y) \otimes F(X) \otimes F(Y)] \rightarrow F(X \vee Y),
$$

such that the following natural diagram commutes:

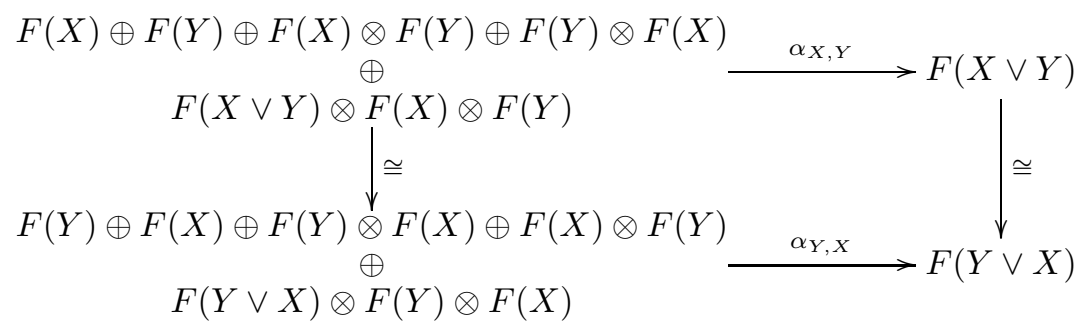

Here the left vertical map takes the summand $F(X)$ to $F(X), F(Y)$ to $F(Y)$, $F(X) \otimes F(Y)$ and $F(Y) \otimes F(X)$ get mapped to $F(X) \otimes F(Y)$ and $F(Y) \otimes F(X)$, respectively, and the map on the last component is the flip map $\tau$. 
Note that using the definition of cross effects (Section 3), the functional equation defining functors of type $\frac{x}{1-x}$ can be rewritten as

$$
F(X) \otimes F(Y) \oplus F(Y) \otimes F(X) \oplus F(X \vee Y) \otimes F(X) \otimes F(Y) \stackrel{\cong}{\Rightarrow} r_{2} F(X, Y) .
$$

Similar restatements are true for all the other functors to be considered, and we will refer to both equations as the defining equation of functors of the given type.

Example 5.2. Let $\mathcal{A} s s_{\mathbb{K}}$ be the category of non-unital associative differential graded $\mathbb{K}$-algebras. (Clearly, $\mathcal{A} s s_{\mathbb{K}}$ is naturally isomorphic to the category of $\mathbb{T}_{1}$ algebras in $C h(\mathbb{K})$, where $\mathbb{T}_{1}$ is the triple given by $\mathbb{T}_{1}=\bigoplus_{n \geq 1} X^{\otimes n}$ for all $X \in C h(\mathbb{K})$.) Here, and in future, the subscript 1 refers to the fact that the summation starts at $n=1$ as opposed to $n=0$, and thus the functor is reduced.) Let $U_{\frac{x}{1-x}}$ be the forgetful functor $\mathcal{A} s s_{\mathbb{K}} \rightarrow C h(\mathbb{K})$. We claim that $U_{\frac{x}{1-x}}$ is of type $\frac{x}{1-x}$. Observe, that if $X \vee Y$ is the coproduct of $X$ and $Y$ in $\mathcal{A} s s_{\mathbb{K}}$, then so is $X \oplus Y \oplus X \otimes Y \oplus Y \otimes X \oplus(X \vee Y) \otimes X \otimes Y$. Indeed, a pair of maps $X \rightarrow A$, $Y \rightarrow A$ in $\mathcal{A} s s_{\mathbb{K}}$ induces a unique map from each component of the sum above into $A$ in an obvious fashion. Now using the fact that two different coproducts of the same pair of objects must be isomorphic, we produce the necessary isomorphisms $\alpha_{X, Y}$.

As in the case of exponential functors we have a lemma.

Lemma 5.3. Every functor $F$ of type $\frac{x}{1-x}$ from $\mathcal{C}$ to $C h(\mathbb{K})$ can be factored as $F=U_{\frac{x}{1-x}} \circ A$, where $A$ is a reduced coproduct preserving functor from $\mathcal{C}$ to $\mathcal{A} s s_{\mathbb{K}}$.

Proof. Define $\mu: F(X) \otimes F(X) \rightarrow F(X)$ as the composite

$$
\begin{aligned}
F(X) & \otimes F(X) \stackrel{i}{\rightarrow} F(X) \oplus F(X) \oplus[F(X) \otimes F(X)] \\
& \oplus[F(X) \otimes F(X)] \oplus[F(X \vee X) \otimes F(X) \otimes F(X)] \stackrel{\alpha_{X, X}}{\rightarrow} F(X \vee X) \stackrel{F(+)}{\rightarrow} F(X) .
\end{aligned}
$$

Here the first map $i$ is the obvious inclusion map. Of course we have two choices for it, and we simply fix one. The two choices reflect the fact that any associative algebra $A$ admits two not necessarily isomorphic multiplications defining algebras $A$ and $A^{o p}$. Naturally, the only thing we need to worry about is being consistent in our choices. The last map $F(+)$ is the functor $F$ applied to the addition map $+: X \vee X \rightarrow X$.

Our next task is to show that $\mu$ is associative.

As with exponential functors, we have a pair of commutative diagrams:

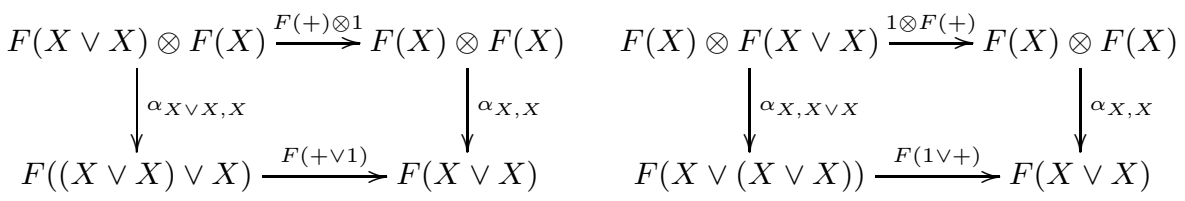

Thus, once again, to prove the associativity, it suffices to show that the diagram

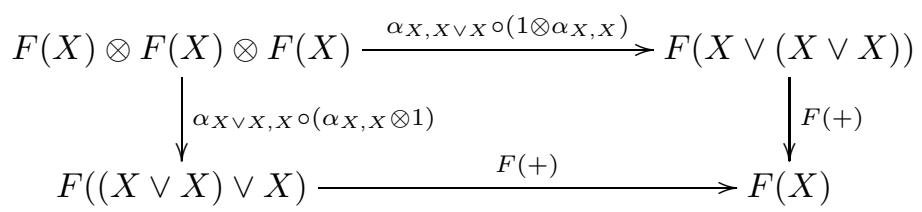

commutes. 
Our strategy is the same as in the case of exponential functors. More precisely, we produce a diagonal map

$$
p: F((X \vee X) \vee X) \rightarrow F(X \vee(X \vee X))
$$

that makes both the top left and bottom right triangles of Diagram (3) commute. In other words, we produce a map $p$ induced by a permutation on $X \vee X \vee X$, which makes the following diagram commute:

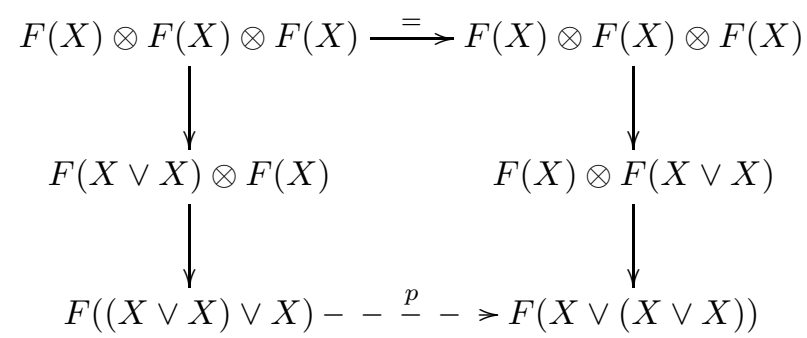

The map $p$ is obtained in a way analogous to the case of exponential functors, i.e. by analyzing the two vertical maps of the above diagram.

To do so, we have to trace through the following (unfortunately very tedious) series of equivalences. The point is that, just as in the exponential case, the left and right vertical composites differ essentially by a permutation of $X \vee X \vee X$.

$3 F(X \vee X) \oplus 4 F(X) \otimes F(X) \otimes F(X) \oplus 2 F(X \vee X) \otimes F(X) \otimes F(X) \otimes F(X) \oplus$ $F(X \vee X \vee X) \otimes F(X \vee X) \otimes F(X) \cong$

$F(X \vee X) \oplus 4 F(X) \oplus 4 F(X) \otimes F(X) \oplus 2 F(X \vee X) \otimes F(X) \otimes F(X) \oplus 4 F(X) \otimes F(X) \otimes$ $F(X) \oplus 2 F(X \vee X) \otimes F(X) \otimes F(x) \otimes F(X) \oplus F(X \vee X \vee X) \otimes F(X \vee X) \otimes F(X) \cong$ $F(X \vee X) \oplus 4 F(X) \oplus 2 F(X \vee X) \otimes F(X) \otimes F(X) \oplus 2 F(X) \otimes[2 F(X) \oplus 2 F(X) \otimes$ $F(X) \oplus F(X \vee X) \otimes F(X) \otimes F(X)] \oplus F(X \vee X \vee X) \otimes F(X \vee X) \otimes F(X) \cong$

$F(X \vee X) \oplus 4 F(X) \oplus 2 F(X \vee X) \otimes F(X) \otimes F(X) \oplus 2 F(X) \otimes F(X \vee X) \oplus$ $F(X \vee X \vee X) \otimes F(X \vee X) \otimes F(X) \cong$

$3 F(X) \oplus 2 F(X \vee X) \otimes F(X) \otimes F(X) \oplus F(X \vee X \vee X)$.

Again it is not hard to see that the map $p$ is the functor $F$ applied to a permutation of $X \vee X \vee X$, hence the two vertical arrows in the above diagram followed by $F(+)$ are equal, which proves that $\mu$ is associative.

To see that the functor into the category of associative algebras that we just defined is coproduct preserving, observe that $F(X \vee Y) \cong$ colim $C_{i}(X, Y)$, where the $C_{i}$ 's are defined recursively:

$$
\begin{gathered}
C_{0}(X, Y)=F(X) \oplus F(Y) \oplus[F(X) \otimes F(Y)] \oplus[F(Y) \otimes F(X)], \\
C_{k+1}(X, Y)=C_{k}(X, Y) \oplus\left[C_{k}(X, Y) \otimes F(X) \otimes F(Y)\right] .
\end{gathered}
$$

Furthermore, clearly each pair of maps $f: F(X) \rightarrow A$ and $g: F(Y) \rightarrow A$ in $\mathcal{A} s s_{\mathbb{K}}$ induces a unique evident map $C_{k}(X, Y) \rightarrow A$ for all $k$. Thus, we obtain a (unique) map from $F(X \vee Y) \cong \operatorname{colim} C_{i}(X, Y)$ to $A$, which proves by universal property of coproducts that $F(X \vee Y)$ is the coproduct of $F(X)$ and $F(Y)$.

\section{TAYLOR TOWERS OF FUNCTORS OF TYPE $\frac{x}{1-x}$}

In calculus, Taylor series of functions cannot always be computed. However, if the function is a solution to a sufficiently 'nice' functional equation, its Taylor series can often be calculated. In this section, we take advantage of the fact that functors of type $\frac{x}{1-x}$ are defined via functional equations to compute their Taylor 
towers. We follow the approach taken by B. Johnson and R. McCarthy in 9] in their treatment of exponential functors. First we will recall some of their results, needed for our computations. For completeness, their results on Taylor towers of exponential functors will also be presented here.

We begin by observing that by Lemma 6.6 of [9], to understand the Taylor tower of functors of type $\frac{x}{1-x}$ we only need to understand the Taylor tower of $U_{\frac{x}{1-x}}$, since if $A$ is a reduced coproduct preserving functor, then

$$
P_{n}(F \circ A) \cong\left(P_{n} F\right) \circ A .
$$

We list two results (Proposition 5.9 and Corollary 5.11 from 9]) that are instrumental in our computations.

Proposition 6.1. For a functor $F: \mathcal{C} \rightarrow C h(\mathbb{K})$, there is a natural isomorphism of $(n+1)$-multifunctors

$\nabla^{n} F\left(X_{1}, \cdots, X_{n} ; Y\right) \cong D_{1}^{(n)}\left[c r_{n+1} F(-, \cdots,-, Y) \oplus c r_{n} F(-, \cdots,-)\right]\left(X_{1}, \cdots, X_{n}\right)$, where the symbol $D_{1}^{(n)}$ indicates that we are applying $D_{1}$ to each variable of the $n$-multifunctor separately, i.e. by holding all but one variable constant at all times.

We do not present the definition of $\nabla^{n} F$ in this document, as the description of $\nabla^{n} F$ provided by this proposition is sufficient for our purposes. The functor $\nabla F$ plays a role analogous to that of the notion of a differential in classical calculus. The following corollary explains our interest in this functor.

Corollary 6.2. For any functor $F: \mathcal{C} \rightarrow C h(\mathbb{K})$,

$$
D_{n} F \simeq \nabla^{n} F(-, \cdots,-; 0)_{h \Sigma_{n}} .
$$

Our objective is to describe the layers $D_{n}$ of the functor $U_{\frac{x}{1-x}}$ in terms of $D_{1}$. For the exponential functor $U_{e}$ this has been done in [9], where the authors computed the cross effects of $U_{e}$ and used the two results above to conclude that

$$
D_{n} U_{e} \simeq\left(\bigotimes_{1}^{n} U_{e}\right)_{h \Sigma_{n}}
$$

Unfortunately, for the case of the functor $U_{\frac{x}{1-x}}$, computing the cross effects proved impractical. However, we are still able to get a formula analogous to Equation (4). First we demonstrate how to do it for $D_{2}$. By Proposition 6.1

$$
\begin{aligned}
& \nabla^{2} U_{\frac{x}{1-x}}\left(X_{1}, X_{2} ; Y\right) \\
& \quad \cong D_{1}^{(2)}\left[c r_{3} U_{\frac{x}{1-x}}(-,-, Y) \oplus c r_{2} U_{\frac{x}{1-x}}(-,-)\right]\left(X_{1}, X_{2}\right) \\
& \quad \cong D_{1}^{(2)}\left[c r_{3} U_{\frac{x}{1-x}}(-,-, Y)\right]\left(X_{1}, X_{2}\right) \oplus D_{1}^{(2)}\left[c r_{2} U_{\frac{x}{1-x}}(-,-)\right]\left(X_{1}, X_{2}\right) .
\end{aligned}
$$

While computing $c r_{3} U_{\frac{x}{1-x}}$ is not impossible, it is easily seen to produce long and tedious formulas which are hard to manage. Naturally, the higher cross effects which will be needed for the general case, are even worse. We make a few observations to simplify the computations.

Remark 6.3. Recall that our intention is to apply Corollary 6.2 to describe $D_{2}$. Consequently, we are interested in computing $D_{1}^{(2)}\left[\operatorname{cr}_{3} U_{\frac{x}{1-x}}\left(X_{1}, X_{2}, Y\right)\right]$ only for the special case $Y=0 . \operatorname{cr}_{3} U_{\frac{x}{1-x}}\left(X_{1}, X_{2}, Y\right)$ is defined via the identity

$$
c r_{3} U_{\frac{x}{1-x}}\left(X_{1}, X_{2}, Y\right) \oplus c r_{2} U_{\frac{x}{1-x}}\left(X_{1}, Y\right) \oplus c r_{2} U_{\frac{x}{1-x}}\left(X_{2}, Y\right) \cong c r_{2} U_{\frac{x}{1-x}}\left(X_{1} \vee X_{2}, Y\right)
$$


It is immediate from the functional equation defining functors of type $\frac{x}{1-x}$ that the second and third terms on the left as well as the term on the right are 0 when $Y=0$. Hence, for $Y=0, c r_{3} U_{\frac{x}{1-x}}\left(X_{1}, X_{2}, Y\right) \cong 0$. Thus, for $Y=0$, $D_{1}^{(2)}\left[\operatorname{cr}_{3} U_{\frac{x}{1-x}}(-,-, Y)\right]\left(X_{1}, X_{2}\right) \cong 0$.

For our next observation, we need yet another result from [9]. It is listed there as Lemma 3.3.

Lemma 6.4. Let $G: \mathcal{C}^{\times n} \rightarrow C h(\mathbb{K})$ be an $n$-multireduced functor, i.e. $G$ is such that $G\left(M_{1}, \cdots, M_{n}\right) \cong 0$ whenever any $M_{i}=0$. Then for $k<n, P_{k} G^{\triangle} \simeq 0$, where $G^{\triangle}$ is $G$ composed with the diagonal $\mathcal{C} \rightarrow \mathcal{C}^{\times n}$. Consequently, for $k<n$, $D_{k} G^{\triangle} \simeq 0$.

Remark 6.5. Suppose $F: \mathcal{C} \rightarrow C h(\mathbb{K})$ is a reduced functor. Consider the functor

$$
H=A \otimes F(X) \otimes B \otimes F(X) \otimes C,
$$

where $A, B, C$ are objects in $C h(\mathbb{K})$. Then $H$ can be viewed as a functor $G: \mathcal{C} \times \mathcal{C} \rightarrow$ $C h(\mathbb{K})$ composed with the diagonal. Since $F$ is reduced, $G$ is 2 -multireduced. Hence by the above Lemma 6.4, $D_{1} H=D_{1} G^{\triangle} \simeq 0$.

By Remark 6.3 to understand $D_{2} U_{\frac{x}{1-x}}$, it is enough to consider

$$
D_{1}^{(2)}\left[c r_{2} U_{\frac{x}{1-x}}(-,-)\right]\left(X_{1}, X_{2}\right)
$$

Recall our formula for $c r_{2} U_{\frac{x}{1-x}}$ :

$$
\begin{aligned}
c r_{2} U_{\frac{x}{1-x}}\left(X_{1}, X_{2}\right) \cong & U_{\frac{x}{1-x}}\left(X_{1}\right) \otimes U_{\frac{x}{1-x}}\left(X_{2}\right) \oplus U_{\frac{x}{1-x}}\left(X_{2}\right) \otimes U_{\frac{x}{1-x}}\left(X_{1}\right) \\
& \oplus U_{\frac{x}{1-x}}\left(X_{1} \vee X_{2}\right) \otimes U_{\frac{x}{1-x}}\left(X_{1}\right) \otimes U_{\frac{x}{1-x}}\left(X_{2}\right) \\
\cong & U_{\frac{x}{1-x}}\left(X_{1}\right) \otimes U_{\frac{x}{1-x}}\left(X_{2}\right) \oplus U_{\frac{x}{1-x}}\left(X_{2}\right) \otimes U_{\frac{x}{1-x}}\left(X_{1}\right) \\
& \oplus\left[U_{\frac{x}{1-x}}\left(X_{1}\right) \oplus U_{\frac{x}{1-x}}\left(X_{2}\right)\right. \\
& \oplus U_{\frac{x}{1-x}}\left(X_{1}\right) \otimes U_{\frac{x}{1-x}}\left(X_{2}\right) \oplus U_{\frac{x}{1-x}}\left(X_{2}\right) \otimes U_{\frac{x}{1-x}}\left(X_{1}\right) \\
& \left.\oplus U_{\frac{x}{1-x}}\left(X_{1} \vee X_{2}\right) \otimes U_{\frac{x}{1-x}}\left(X_{1}\right) \otimes U_{\frac{x}{1-x}}\left(X_{2}\right)\right] \\
& \otimes U_{\frac{x}{1-x}}\left(X_{1}\right) \otimes U_{\frac{x}{1-x}}\left(X_{2}\right) .
\end{aligned}
$$

To get the second equivalence we used the defining equation of functors of type $\frac{x}{1-x}$ again. Thus, all the terms except $U_{\frac{x}{1-x}}\left(X_{1}\right) \otimes U_{\frac{x}{1-x}}\left(X_{2}\right)$ and $U_{\frac{x}{1-x}}\left(X_{2}\right) \otimes U_{\frac{x}{1-x}}\left(X_{1}\right)$ have at least two factors of $U_{\frac{x}{1-x}}\left(X_{1}\right)$ or two factors of $U_{\frac{x}{1-x}}\left(X_{2}\right)$. Consequently by Remark 6.5, these terms vanish after we apply either $D_{1}^{1}$ or $D_{1}^{2}$, where $D_{1}^{i}$ is $D_{1}$ applied to the $i$ 'th variable with the other variables held constant. Hence,

$$
\begin{aligned}
D_{1}^{(2)}\left[c r_{2} U_{\frac{x}{1-x}}(-,-)\right]\left(X_{1}, X_{2}\right) \cong & D_{1}\left[U_{\frac{x}{1-x}}\left(-{ }_{1}\right) \otimes U_{\frac{x}{1-x}}\left(-_{2}\right)\right. \\
& \left.\oplus U_{\frac{x}{1-x}}(-2) \otimes U_{\frac{x}{1-x}}\left(--_{1}\right)\right]\left(X_{1}, X_{2}\right) \\
\cong & D_{1} U_{\frac{x}{1-x}}(-)\left(X_{1}\right) \otimes D_{1} U_{\frac{x}{1-x}}(-)\left(X_{2}\right) \\
& \oplus D_{1} U_{\frac{x}{1-x}}(-)\left(X_{2}\right) \otimes D_{1} U_{\frac{x}{1-x}}(-)\left(X_{1}\right) .
\end{aligned}
$$

By Corollary 6.2, we conclude that

$$
D_{2} U_{\frac{x}{1-x}}(-)(X) \cong D_{1} U_{\frac{x}{1-x}}(-)(X) \otimes D_{1} U_{\frac{x}{1-x}}(-)(X)
$$


For the general case $D_{n}$, first we note that by induction $c r_{n+1} U_{\frac{x}{1-x}}\left(X_{1}, \cdots, X_{n}, Y\right)$ can be written as a sum of terms each of which contains at least one factor of $U_{\frac{x}{1-x}}(Y)$. Hence, as in Remark 6.3 for $Y=0$,

$$
D_{1}^{(n)}\left[c r_{n+1} U_{\frac{x}{1-x}}(-, \cdots,-, Y)\right]\left(X_{1}, \cdots, X_{n}\right) \simeq 0 .
$$

Thus, we have to worry only about

$$
D_{1}^{(n)}\left[c r_{n} U_{\frac{x}{1-x}}(-, \cdots,-)\right]\left(X_{1}, \cdots, X_{n}\right) .
$$

Observe that, analogous to the case $n=2$, the only terms of $c r_{n} U_{\frac{x}{1-x}}\left(X_{1}, \cdots, X_{n}\right)$ that do not contain at least two factors of $U_{\frac{x}{1-x}}\left(X_{i}\right)$ for some $i$ are those of the form $U_{\frac{x}{1-x}}\left(X_{\sigma(1)}\right) \otimes \cdots \otimes U_{\frac{x}{1-x}}\left(X_{\sigma(n)}\right)$, where $\sigma$ is any element of the permutation group $\Sigma_{n}$. Hence by Remark 6.5 these are the only (possibly) non-vanishing terms after we apply $D_{1}^{(n)}$. By Corollary 6.2, we are entitled to conclude that

$$
D_{n} U_{\frac{x}{1-x}} \cong \bigotimes^{n} D_{1} U_{\frac{x}{1-x}} .
$$

\section{LOGARITHMIC FUNCTORS AND THEIR TAYLOR TOWERS}

From now on we will assume that $\mathbb{K}$ is a field of char 0 .

In this section, we continue our investigation of functors patterned after elementary functions of one variable by considering logarithmic functions $-\log (1-a x)$. While it is a good candidate for our purposes, as it has a relatively simple Taylor series and is easily seen to be related to a common algebraic structure, we have yet another reason for singling out logarithmic functors.

To explain our interest we refer to the functions of real variables again. Observe that $f(x)=\frac{1}{1-x}-1$ and $f(x)=e^{x}-1$ are respectively the first element and the limit of the sequence of functions

$$
f_{n}(x)=\left(1-\frac{x}{2^{n-1}}\right)^{-2^{n-1}}-1 .
$$

Of course, here any number greater than 1 can be used instead of 2 . This is reminiscent of the situation with the homology of little $n$-cubes operads $e_{n}$, where $e_{n}$ for $n>1$ interpolate between $e_{1}$ and $e_{\infty}$, which produce associative and commutative algebras respectively, much like the exponential functors and functors of type $\frac{x}{1-x}$. Hence the hope that the functors associated with the functions $f_{n}$ will provide an insight in the algebras over the homology of little $n$-cubes operads.

However, before we attempt to pattern a class of functors after the functions $f_{n}$ above, we note that $f_{n}(x)=e^{-2^{n-1} \log \left(1-\frac{x}{2^{n-1}}\right)}-1$. Thus, the function $-\log (1-x)$, in addition to being of interest on its own, is closely related to $f_{n}$.

Before proceeding, we pause to observe that the suspension functor $\Sigma$ behaves like multiplication by $1 / 2$ (or any other number less than one) for the following two reasons. First, both are linear. Second, repeatedly multiplying by $1 / 2$ creates a sequence converging to 0 . Analogously, applying $\Sigma$ increases the connectivity, thus taking us 'closer' to a contractible object. We also note that multiplication by 2 is represented by desuspension $\Sigma^{-1}$ since it is the inverse operation of $\Sigma$.

As before, the following definition is inspired by the fact that $-\log (1-a x)$ are the only real variable analytic functions satisfying the functional equation

$$
f\left(\frac{\sqrt{a} x}{1-a x} \cdot \frac{\sqrt{a} y}{1-a y}\right)+f(x)+f(y)=f(x+y) .
$$


For the simpler (but illustrative) case of the function $-\log (1-x)$ we get the functional equation

$$
f\left(\frac{x}{1-x} \cdot \frac{y}{1-y}\right)+f(x)+f(y)=f(x+y),
$$

and simple arithmetic manipulations illustrate that the term $\frac{x}{1-x}$ in the above equation appears due to the simple identity

$$
\left(e^{x}-1\right) \circ(-\log (1-x))=\frac{x}{1-x} .
$$

Definition 7.1. A reduced functor $F: C h(\mathbb{K}) \rightarrow C h(\mathbb{K})$ is logarithmic if there is a natural isomorphism

$$
\alpha_{X, Y}: F(U X \otimes U Y) \oplus F(X) \oplus F(Y) \rightarrow F(X \vee Y),
$$

where (motivated by Identity (5) $U=\mathbb{P}_{1} F$ and is a functor of type $\frac{x}{1-x}$ (here $\mathbb{P}_{1}$ is the non-unital version of the symmetric algebra triple $\left.\mathbb{P}\right)$, such that for all $X, Y \in C h(\mathbb{K})$ the natural diagram commutes:

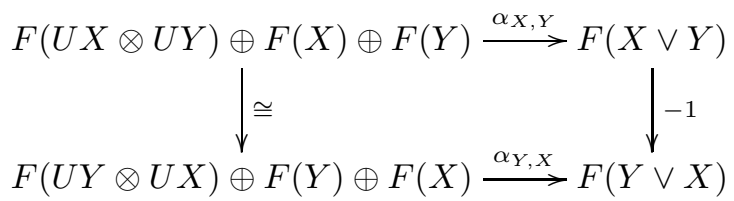

Shortly we will make additional comments on our choice of the particular form of functor $U$ (e.g. see Example [7.3).

Before discussing the logarithmic functors, we digress a little to recall the definition of a Lie algebra over chain complexes (i.e. in graded context). The reason for introducing the Lie algebras at this point will be evident from Example 7.3 In particular, the following definition explains our choice (of -1) of the right-hand side vertical map in the above diagram.

Definition 7.2. $A \in C h(\mathbb{K})$ is a Lie algebra if it is equipped with a bracket operation $[-,-]: A \otimes A \rightarrow A$, such that the composites $(A \otimes A)^{C_{2}} \rightarrow A \otimes A \rightarrow A$ and $(A \otimes A \otimes A)^{C_{3}} \rightarrow A \otimes A \otimes A \rightarrow A$ are zero. Here $C_{2}$ and $C_{3}$ are the cyclic groups of size 2 and 3 respectively, $A^{G}$ denotes the fixed points of the action of $G$ on $A$, and the actions of $C_{2}$ and $C_{3}$ are defined as follows:

The non-trivial element of $C_{2}$ acts on $A \otimes A$ by taking $a \otimes b$ to $(-1)^{|a||b|} b \otimes a$, and the two non-trivial elements of $C_{3}$ act by mapping $a \otimes b \otimes c$ to $(-1)^{|a||c|+|b||c|} c \otimes a \otimes b$ and $(-1)^{|a||c|+|a||b|} b \otimes c \otimes a$.

Example 7.3. Let $\mathbb{L}$ be the free Lie algebra functor, i.e. the left adjoint to the forgetful functor $U_{\log }$ from the category $\mathcal{L}_{\mathbb{K}}$ of Lie algebras to $C h(\mathbb{K})$. Alternatively, $\mathbb{L}(M)$ can be defined as the Lie subalgebra of the Lie algebra Lie $(\mathbb{T}(M))$ underlying the tensor algebra $\mathbb{T}(M)$, generated by $M$. We claim that $F=U_{\log } \circ \mathbb{L}$ is a logarithmic functor. First observe that, in characteristic 0, by the Poincaré-Birkhoff-Witt Theorem, $\mathbb{P}_{1} F \cong \mathbb{T}_{1}$, where $\mathbb{P}_{1}$ and $\mathbb{T}_{1}$ are the non-unital versions of the symmetric algebra triple $\mathbb{P}$ and the tensor algebra triple $\mathbb{T}$, respectively. Now it is not hard to see that the functor satisfies the equation

$$
c r_{2} U_{\log } \circ \mathbb{L}(X, Y) \cong U_{\log } \circ \mathbb{L}\left(\mathbb{T}_{1} X \otimes \mathbb{T}_{1} Y\right) .
$$


Indeed, one simply needs to verify that there is an isomorphism of underlying modules

$$
\mathbb{L}\left(\mathbb{T}_{1} X \otimes \mathbb{T}_{1} Y\right) \oplus \mathbb{L}(X) \oplus \mathbb{L}(Y) \cong \mathbb{L}(X \vee Y),
$$

which is easily accomplished by comparison of the generator of the two sides.

As a curious point, it is worth noting that the Poincaré-Birkhoff-Witt Theorem is a restatement of the simple functional identity

$$
e^{-\log (1-x)}-1=\frac{x}{1-x}
$$

Remark 7.4. Note that Example 7.3 gives rise to an entire family of examples. In essence, logarithmic functors are defined by describing the action of the functor on coproducts of objects (or equivalently, by describing the second cross effect of the functor) in terms of the functor evaluated at the objects themselves.

Consequently, if we precompose a functor which is logarithmic with any coproduct-preserving functor, the resulting functor, once again, will be logarithmic.

Remark 7.5. As it is the case with exponential functors and functors of type $\frac{x}{1-x}$, Example 7.3 is, in essence, the only example of logarithmic functors, that is, Example 7.3. combined with Remark 7.4, produces all the examples of logarithmic functors. In other words, there is an analogue of Lemma 4.3 and Lemma 5.3 for the case of logarithmic functors, stating that all logarithmic functors differ from the above example by a coproduct-preserving functor. However, the proof of this result is rather tedious, and since the result is not essential for any of the computations in the remainder of the paper, we forgo both stating the result (in the form of a lemma) and presenting the proof for the sake of economy and compactness.

It is worth noting that this remark also applies to the future case of functors of type $f_{n}$ which are to be treated in Section 8 Once again, the deriving triple of $n$-Poisson algebras will be in essence the only example of functors of type $f_{n}$.

As with functors of type $\frac{x}{1-x}$, our next goal is to express the higher derivatives of logarithmic functors in terms of $D_{1}$. Again, this is an application of the theory developed by B. Johnson and R. McCarthy in 9].

Let $F$ be a logarithmic functor. We begin with the computation of $D_{2} F$. By Proposition 6.1,

$$
\begin{aligned}
\nabla^{2} F\left(X_{1}, X_{2} ; Y\right) & \cong D_{1}^{(2)}\left[c r_{3} F(-,-, Y) \oplus c r_{2} F(-,-)\right]\left(X_{1}, X_{2}\right) \\
& \cong D_{1}^{(2)}\left[c r_{3} F(-,-, Y)\right]\left(X_{1}, X_{2}\right) \oplus D_{1}^{(2)}\left[c r_{2} F(-,-)\right]\left(X_{1}, X_{2}\right) .
\end{aligned}
$$

Recall that

$$
c r_{3} F\left(X_{1}, X_{2}, Y\right) \oplus c r_{2} F\left(X_{1}, Y\right) \oplus c r_{2} F\left(X_{2}, Y\right) \cong c r_{2} F\left(X_{1} \vee X_{2}, Y\right) .
$$

However, $D_{1}^{(2)} \mathrm{cr}_{2} F\left(X_{i}, Y\right)=0$ for $i=1,2$, since $\mathrm{cr}_{2} F\left(X_{i}, Y\right)=0$ is constant with respect to $X_{1}$ or $X_{2}$. Hence,

$$
\begin{aligned}
D_{1}^{(2)}\left[c r_{3} F(-,-, Y)\right]\left(X_{1}, X_{2}\right) & \cong D_{1}^{(2)}\left[c r_{2} F(-\vee-, Y)\right]\left(X_{1}, X_{2}\right) \\
& \cong D_{1}^{(2)}[F(U(-\vee-) \otimes U Y)]\left(X_{1}, X_{2}\right) .
\end{aligned}
$$

Of course, our intention is to apply Corollary 6.2, and hence we are interested only in the case $Y=0$. Observe that since the functors $A \otimes-, U$ and $F$ are reduced, $D_{1}^{(2)}[F(U(-\vee-) \otimes U Y)]\left(X_{1}, X_{2}\right) \cong 0$ for $Y=0$. Thus, we only need to compute $D_{1}^{(2)}\left[c r_{2} F(-,-)\right]\left(X_{1}, X_{2}\right)$. 
To do this we use the result listed as Lemma 5.7 in [9] which establishes a chain rule for functors analogous to the chain rule for functions of one variable.

Lemma 7.6. Let $\mathcal{A}$ and $\mathcal{A}^{\prime}$ be Abelian categories, and let functors $F$ and $G$ be such that $G: \mathcal{C} \rightarrow \mathcal{A}^{\prime}$ and $F: \mathcal{A}^{\prime} \rightarrow C h \mathcal{A}$. Then

$$
D_{1}(F \circ G) \cong D_{1} F \circ D_{1} G \text {. }
$$

We use this lemma to compute the differential $D_{1}^{(2)}$ of $c r_{2} F$ :

$$
\begin{aligned}
D_{1} c r_{2} F\left(-, X_{2}\right)\left(X_{1}\right) & \cong D_{1} F\left(U(-) \otimes U X_{2}\right)\left(X_{1}\right) \cong D_{1}\left[F \circ\left(U(-) \otimes U X_{2}\right)\right]\left(X_{1}\right) \\
& \cong D_{1} F \circ D_{1}\left[U(-) \otimes U X_{2}\right]\left(X_{1}\right) \\
& \cong D_{1} F \circ\left[D_{1} U(-)\left(X_{1}\right) \otimes U X_{2}\right] \\
& \cong\left[D_{1} U(-)\left(X_{1}\right) \otimes U X_{2}\right] \otimes D_{1} F(\mathbb{K}),
\end{aligned}
$$

where $\mathbb{K}$ is the ground field. The last equivalence holds by Lemma 3.3 because $D_{1} F$ is linear. Furthermore, note that

$$
D_{1} U(-)\left(X_{1}\right) \cong D_{1}\left(\mathbb{P}_{1} F\right) U(-)\left(X_{1}\right) \cong D_{1} \mathbb{P}_{1} \circ D_{1} F(-)\left(X_{1}\right) \cong D_{1} F(-)\left(X_{1}\right) .
$$

Combining these calculations we have that

$$
D_{1} c r_{2} F\left(-, X_{2}\right)\left(X_{1}\right) \cong D_{1} F\left(X_{1}\right) \otimes U\left(X_{2}\right) \otimes D_{1} F(\mathbb{K}) .
$$

Consequently, using the linearity of $D_{1}$ again, we get

$$
\begin{aligned}
D_{1}^{(2)} c r_{2} F(-,-)\left(X_{1}, X_{2}\right) & \cong D_{1}^{2}\left[D_{1} F\left(X_{1}\right) \otimes U(-) \otimes D_{1} F(\mathbb{K})\right]\left(X_{2}\right) \\
& \cong D_{1} F\left(X_{1}\right) \otimes D_{1} U(-)\left(X_{2}\right) \otimes D_{1} F(\mathbb{K}) \\
& \cong D_{1} F\left(X_{1}\right) \otimes D_{1} F\left(X_{2}\right) \otimes D_{1} F(\mathbb{K}) .
\end{aligned}
$$

Here the superscript in $D_{1}^{2}$ refers to the fact that the linearization functor $D_{1}$ is applied with respect to the second variable of the two-variable functor $\mathrm{Cr}_{2} F(-,-)$. Thus, we have completed the computation of $\nabla^{2} F(X, X ; 0)$ :

$$
\nabla^{2} F(X, X ; 0) \cong D_{1} F\left(X_{1}\right) \otimes D_{1} F\left(X_{2}\right) \otimes D_{1} F(\mathbb{K}) .
$$

Now we consider the general case.

Remark 7.7. Recall that

$$
\begin{aligned}
c r_{n+1} F\left(X_{1}, X_{2}, \cdots, X_{n}, Y\right) & \oplus c r_{n} F\left(X_{2}, \cdots, X_{n}, Y\right) \oplus c r_{n} F\left(X_{1}, X_{3}, \cdots, X_{n}, Y\right) \\
\cong & c r_{n} F\left(X_{1} \vee X_{2}, X_{3}, \cdots, X_{n}, Y\right) .
\end{aligned}
$$

Hence when we apply $D_{1}^{(n)}$ to the two sides of the above equation, the second and the third terms on the left vanish since they are constant with respect to $X_{1}$ and $X_{2}$, respectively. Thus,

$$
\begin{aligned}
& D_{1}^{(n)} c r_{n+1} F(-, \cdots,-, Y)\left(X_{1}, \cdots, X_{n}\right) \\
& \quad \cong D_{1}^{(n)} c r_{n} F(-\vee-,-, \cdots,-, Y)\left(X_{1}, \cdots, X_{n}\right) .
\end{aligned}
$$

As with functors of type $\frac{x}{1-x}$, we are interested in the case $Y=0$ (see Corollary 6.2). By the above arguments,

$$
D_{1}^{(n)} c r_{n} F(-\vee-,-, \cdots,-, Y) \cong D_{1}^{(n)} c r_{n-1} F(-\vee-\vee-, \cdots,-, Y) .
$$


Hence, for $Y=0$, by induction on $n$, we have that

$$
D_{1}^{(n)} \mathrm{cr}_{n} F(-\vee-,-, \cdots,-, Y)\left(X_{1}, \cdots, X_{n}\right) \cong 0 .
$$

Consequently, for $Y=0$

$$
D_{1}^{(n)} c r_{n+1} F(-, \cdots,-, Y)\left(X_{1}, \cdots, X_{n}\right) \cong 0 .
$$

Thus, we only need to understand $D_{1}^{(n)} c r_{n} F(-, \cdots,-)\left(X_{1}, \cdots, X_{n}\right)$. By iterating the argument in Remark 7.7, we get that

$$
\begin{aligned}
D_{1}^{(n)} c r_{n} F(-, \cdots,-)\left(X_{1}, \cdots, X_{n}\right) & \cong D_{1}^{(n)} c r_{2} F(-\vee \cdots \vee-,-)\left(X_{1}, \cdots, X_{n}\right) \\
& \cong D_{1}^{(n)} F(U(-\vee \cdots \vee-) \otimes U(-))\left(X_{1}, \cdots, X_{n}\right) .
\end{aligned}
$$

Recall that $U$ is a functor of type $\frac{x}{1-x}$, and thus using the computations performed in the previous section, by Equation (6), we have that

$$
\begin{aligned}
& D_{1}^{(n-1)} U(-\vee \cdots \vee-)\left(X_{1}, \cdots, X_{n-1}\right) \\
& \quad \simeq \bigoplus D_{1} U(-)\left(X_{\sigma(1)}\right) \otimes \cdots \otimes D_{1} U(-)\left(X_{\sigma(n-1)}\right) \\
& \quad \simeq \bigoplus D_{1} F(-)\left(X_{\sigma(1)}\right) \otimes \cdots \otimes D_{1} F(-)\left(X_{\sigma(n-1)}\right),
\end{aligned}
$$

where the sums on the right are taken over all permutations $\sigma$ of the group $\Sigma_{n-1}$. Combining these calculations with Lemma 7.6 on chain rule, as well as our computation of the second layer $D_{2}$, we get that

$$
\begin{aligned}
D_{1}^{(n)} & c r_{n} F\left(X_{1}, \cdots, X_{n}\right) \\
& \simeq D_{1}^{n}\left[D_{1}^{(n-1)} c r_{n} F(-, \cdots,-,-)\left(X_{1}, \cdots, X_{n-1}\right)\right]\left(X_{n}\right) \\
& \simeq D_{1}^{n}\left[D_{1}^{(n-1)} F(U(-\vee \cdots \vee-) \otimes U(-))\left(X_{1}, \cdots, X_{n-1}\right)\right]\left(X_{n}\right) \\
& \simeq\left[\bigoplus D_{1} F(-)\left(X_{\sigma(1)}\right) \otimes \cdots \otimes D_{1} F(-)\left(X_{\sigma(n-1)}\right)\right] \otimes D_{1}^{n}\left[D_{1} F(\mathbb{K} \otimes U(-))\right]\left(X_{n}\right) \\
& \simeq\left[\bigoplus D_{1} F(-)\left(X_{\sigma(1)}\right) \otimes \cdots \otimes D_{1} F(-)\left(X_{\sigma(n-1)}\right)\right] \otimes D_{1} F\left(X_{n}\right) \otimes D_{1} F(\mathbb{K}) .
\end{aligned}
$$

Hence, we are allowed to conclude that

$$
\nabla^{n} F(X, \cdots, X ; 0) \cong \bigoplus\left[D_{1} F(X) \otimes \cdots \otimes D_{1} F(X)\right] \otimes D_{1} F(X) \otimes D_{1} F(\mathbb{K}),
$$

where there are $n-1$ factors in the brackets of the sum on the right, and the sum is again taken over all permutations of the group $\Sigma_{n-1}$.

Hence for $n>1$, by Corollary 6.2 $D_{n} F$ is the tensor product over $\Sigma_{n}$ of the $n$ 'th tensor power of $D_{1} F$ with an $n-1$ dimensional representation of $\Sigma_{n}$.

We pause here to recall the Lie representation Lie $(n)$ of $\Sigma_{n}$. We refer to 15 for details, as this paragraph is almost entirely from there. If we denote by $\mathcal{L}_{n}$ the free Lee algebra on $n$ generators, then $\operatorname{Lie}(n)$ is the so-called multilinear part of $\mathcal{L}_{n}$, that is, $\operatorname{Lie}(n)$ is the direct summand of $\mathcal{L}_{n}$ spanned by all Lee monomial where each of the generators appears precisely once. Lie $(n)$ is the $n$th space of the Lie operad. Note that Lie $(n)$ is a free $\mathbb{K}$-module of rank $(n-1)$ !. Moreover, the symmetric group $\Sigma_{N}$ acts on $\operatorname{Lie}(n)$ simply by permuting the $n$ generators. In addition, the left regulated brackets

$$
\sigma \cdot\left[x_{1},\left[x_{2}, \cdots,\left[x_{n-1}, x_{n}\right] \cdots\right]\right]
$$

for $\sigma \in \Sigma_{n-1}$ form a basis of $\operatorname{Lie}(n)$. 
Returning to our computations of differentials of logarithmic functors, we observe that by virtue of simple comparison with $\operatorname{Lie}(n)$, we are allowed to conclude that

$$
D_{n} F \cong \operatorname{Lie}(n) \otimes_{h \Sigma_{n}} \bigotimes^{n} D_{1} F U \otimes D_{1} F(\mathbb{K}),
$$

where $\operatorname{Lie}(n)$ is the Lie representation of $\Sigma_{n}$.

\section{FunCtORS OF TYPE $f_{n}$}

Now we get back to functors of type $f_{n}$ for $n>1$. The functional equation (for reduced analytic functions) defining $f_{n}(x)$ is as follows:

$$
\begin{gathered}
f(x+y)=f(x)+f(y)+f(x) f(y)+f\left(2^{n-1} \cdot \frac{x / 2^{n-1}}{1-x / 2^{n-1}} \cdot \frac{y / 2^{n-1}}{1-y / 2^{n-1}}\right) \\
+f\left(2^{n-1} \cdot \frac{x / 2^{n-1}}{1-x / 2^{n-1}} \cdot \frac{y / 2^{n-1}}{1-y / 2^{n-1}}\right) \cdot[f(x)+f(y)+f(x) f(y)] .
\end{gathered}
$$

Naturally, the more general case $f_{n}(a x)$ is defined by a similar, if somewhat more messy, equation.

To verify this functional equation, first define $g(x)=\left(1-\frac{x}{2^{n-1}}\right)^{-2^{n-1}}$, i.e. $g(x)=$ $f(x)+1$. Note that in terms of $g(x)$ 's and $g(y)$ 's the right-hand side of the functional equation is

$$
\begin{aligned}
g(x) & -1+g(y)-1+g(x) g(y)-g(x)-g(y)+1 \\
& +g\left(2^{n-1} \cdot \frac{x / 2^{n-1}}{1-x / 2^{n-1}} \cdot \frac{y / 2^{n-1}}{1-y / 2^{n-1}}\right)-1 \\
& +\left[g\left(2^{n-1} \cdot \frac{x / 2^{n-1}}{1-x / 2^{n-1}} \cdot \frac{y / 2^{n-1}}{1-y / 2^{n-1}}\right)-1\right][g(x) g(y)-1] \\
& =g(x) g(y)-1+g\left(2^{n-1} \cdot \frac{x / 2^{n-1}}{1-x / 2^{n-1}} \cdot \frac{y / 2^{n-1}}{1-y / 2^{n-1}}\right)-1 \\
& +\left[g\left(2^{n-1} \cdot \frac{x / 2^{n-1}}{1-x / 2^{n-1}} \cdot \frac{y / 2^{n-1}}{1-y / 2^{n-1}}\right)-1\right][g(x) g(y)-1] \\
& =g\left(2^{n-1} \cdot \frac{x / 2^{n-1}}{1-x / 2^{n-1}} \cdot \frac{y / 2^{n-1}}{1-y / 2^{n-1}}\right) \cdot g(x) g(y)-1 \\
& =\left[\left(1-\frac{x / 2^{n-1}}{1-x / 2^{n-1}} \cdot \frac{y / 2^{n-1}}{1-y / 2^{n-1}}\right) \cdot\left(1-x / 2^{n-1}\right)\left(1-y / 2^{n-1}\right)\right]^{-2^{n-1}}-1 \\
& =\left[1-\frac{x}{2^{n-1}}-\frac{y}{2^{n-1}}\right]^{-2^{n-1}}-1=g(x+y)-1=f(x+y) .
\end{aligned}
$$

Recalling that multiplication by 2 corresponds to desuspension, we give the following definition.

Definition 8.1. A reduced functor $F: C h(\mathbb{K}) \rightarrow C h(\mathbb{K})$ is of type $f_{n}$ if there is a natural isomorphism

$$
\begin{aligned}
\alpha_{X, Y} & : F(X) \oplus F(Y) \oplus F(X) \otimes F(Y) \\
& \oplus F\left(\Sigma^{-n+1} U X \otimes U Y\right) \\
& \oplus F\left(\Sigma^{-n+1} U X \otimes U Y\right) \otimes[F(X) \oplus F(Y) \oplus F(X) \otimes F(Y)] \rightarrow F(X \vee Y),
\end{aligned}
$$


where $U=\mathbb{P}_{1} \Sigma^{n-1} F$ is a functor of type $\frac{x}{1-x}$, such that the following two diagrams commute:

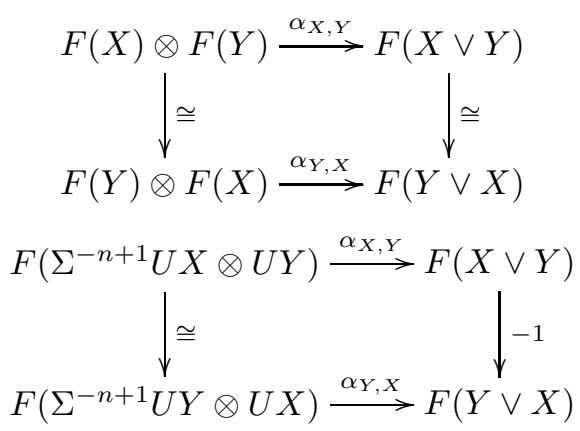

Here we are abusing the notation, as the $\alpha_{X, Y}$ 's and $\alpha_{Y, X}$ 's in the above diagrams are actually inclusions followed by the original $\alpha_{X, Y}$ 's and $\alpha_{Y, X}$ 's.

Note that the ingredients of the functional equation defining the functors of type $f_{n}$ are familiar. In particular, the term $F\left(\Sigma^{-n+1} U X \otimes U Y\right)$ resembles the defining equation of logarithmic functors with a shift, while the term $F(X) \otimes F(Y)$ is the key component of exponential functors. Hence it is reasonable to expect that examples of functors of type $f_{n}$ evaluated at an object $X$ are equipped with both a Lie bracket and a commutative multiplication.

We make a few observations before we can produce examples of functors of type $f_{n}$.

Remark 8.2. The purpose of this remark is to observe that if $L$ is a logarithmic functor, then $\mathbb{P} \Sigma^{-n+1} L \Sigma^{n-1} X$ is equipped with a Lie bracket of degree $n-1$. In particular, we produce a map

$$
[-,-]_{n-1}: \mathbb{P}\left(\Sigma^{1-n} L\left(\Sigma^{n-1} X\right)\right) \otimes \mathbb{P}\left(\Sigma^{1-n} L\left(\Sigma^{n-1} X\right)\right) \rightarrow \Sigma^{1-n} \mathbb{P}\left(\Sigma^{1-n} L\left(\Sigma^{n-1} X\right)\right) .
$$

A typical summand on the left is of the form

$$
\left[\Sigma^{1-n} L\left(\Sigma^{n-1} X\right)\right]^{\otimes l} / \Sigma_{l} \otimes\left[\Sigma^{1-n} L\left(\Sigma^{n-1} X\right)\right]^{\otimes k} / \Sigma_{k} .
$$

So to describe (7), it is enough to produce the maps

$$
\begin{gathered}
{\left[\Sigma^{1-n} L\left(\Sigma^{n-1} X\right)\right]^{\otimes l} / \Sigma_{l} \otimes\left[\Sigma^{1-n} L\left(\Sigma^{n-1} X\right)\right]^{\otimes k} / \Sigma_{k}} \\
\rightarrow \Sigma^{1-n}\left[\Sigma^{1-n} L\left(\Sigma^{n-1} X\right)\right]^{\otimes k+l-1} / \Sigma_{k+l-1} .
\end{gathered}
$$

First, since $L$ is logarithmic, we have the bracket $[-,-]_{L}: L\left(\sum^{n-1} X\right) \otimes L\left(\sum^{n-1} X\right)$

$\rightarrow L\left(\sum^{n-1} X\right)$, which (by desuspending $2(n-1)$ times) produces a map

$$
\Sigma^{1-n} L\left(\Sigma^{n-1} X\right) \otimes \Sigma^{1-n} L\left(\Sigma^{n-1} X\right) \rightarrow \Sigma^{1-n}\left(\Sigma^{1-n} L\left(\Sigma^{n-1} X\right)\right)
$$

(see Remark 7.51). As stated, our objective is to extend this map to terms described by Equation (8). This will be done in two steps. First we consider the case $l=1$. To begin, we make a general observation.

Fix a chain complex $C$. Consider the map

$$
C^{\otimes k} \rightarrow \bigoplus_{1}^{k}\left[C \otimes C^{\otimes(k-1)} / \Sigma_{k-1}\right] \stackrel{+}{\rightarrow} C \otimes C^{\otimes(k-1)} / \Sigma_{k-1},
$$


where the second map is the obvious addition map, and the first map is defined on an element $a_{1} \otimes \cdots \otimes a_{k} \in C^{\otimes k}$ by

$$
a_{1} \otimes \cdots \otimes a_{k} \mapsto \Sigma_{\sigma} a_{\sigma(1)} \otimes \cdots \otimes a_{\sigma(k)},
$$

with the sum on the right ranging over $k$ cyclic permutations of $k$ letters. Note that this map factors through $C^{\otimes k} / \Sigma_{k}$, thus producing a map $\gamma_{C}: C^{\otimes k} / \Sigma_{k} \rightarrow$ $C \otimes C^{\otimes(k-1)} / \Sigma_{k-1}$.

Returning to our case, observe that we have a sequence of maps

$$
\begin{aligned}
& \Sigma^{1-n} L\left(\Sigma^{n-1} X\right) \otimes\left[\Sigma^{1-n} L\left(\Sigma^{n-1} X\right)\right]^{\otimes k} / \Sigma_{k} \\
& \rightarrow \Sigma^{1-n} L\left(\Sigma^{n-1} X\right) \otimes \Sigma^{1-n} L\left(\Sigma^{n-1} X\right) \otimes\left[\Sigma^{1-n} L\left(\Sigma^{n-1} X\right)\right]^{\otimes k-1} / \Sigma_{k-1} \\
& \stackrel{[-,-] \otimes 1}{\longrightarrow} \Sigma^{1-n}\left(\Sigma^{1-n} L\left(\Sigma^{n-1} X\right)\right) \otimes\left[\Sigma^{1-n} L\left(\Sigma^{n-1} X\right)\right]^{\otimes k-1} / \Sigma_{k-1} \\
& \rightarrow \Sigma^{1-n}\left[\Sigma^{1-n} L\left(\Sigma^{n-1} X\right)\right]^{\otimes k} / \Sigma_{k},
\end{aligned}
$$

where the first map is the map $\gamma_{\Sigma^{1-n} L\left(\Sigma^{n-1} X\right)}$ defined above. The general case follows by induction:

$$
\begin{aligned}
& {\left[\Sigma^{1-n} L\left(\Sigma^{n-1} X\right)\right]^{\otimes l} / \Sigma_{l} \otimes\left[\Sigma^{1-n} L\left(\Sigma^{n-1} X\right)\right]^{\otimes k} / \Sigma_{k}} \\
& \stackrel{\gamma \otimes 1}{\longrightarrow}\left[\Sigma^{1-n} L\left(\Sigma^{n-1} X\right)\right] \otimes l-1 / \Sigma_{l-1} \otimes \Sigma^{1-n} L\left(\Sigma^{n-1} X\right) \otimes\left[\Sigma^{1-n} L\left(\Sigma^{n-1} X\right)\right]^{\otimes k} / \Sigma_{k} \\
& \stackrel{1 \otimes[-,-]}{\longrightarrow}\left[\left(\Sigma^{1-n} L\left(\Sigma^{n-1} X\right)\right)\right]^{\otimes l-1} / \Sigma_{l-1} \otimes \Sigma^{1-n}\left[\Sigma^{1-n} L\left(\Sigma^{n-1} X\right)\right]^{\otimes k} / \Sigma_{k} \\
& \rightarrow \Sigma^{1-n}\left[\Sigma^{1-n} L\left(\Sigma^{n-1} X\right)\right]^{\otimes k+l-1} / \Sigma_{k+l-1} .
\end{aligned}
$$

The map $[-,-]_{n-1}$ we just defined is a Lie bracket, as it is essentially the $n-1$ suspension of the Lie bracket $[-,-]_{L}$.

This prompts us to recall the definition of $n$-Poisson algebras (see [5]).

Definition 8.3. $P \in C h(\mathbb{K})$ is an $(n+1)$-Poisson algebra if it is equipped with a commutative multiplication $\mu: P \otimes P \rightarrow P$ and a bracket $[-,-]: P \otimes P \rightarrow \Sigma^{-n} P$ such that, as with Lie algebras, the composites $(P \otimes P)^{C_{2}} \rightarrow P \otimes P \rightarrow \Sigma^{-n} P$ and $(P \otimes P \otimes P)^{C_{3}} \rightarrow P \otimes P \otimes P \rightarrow P \otimes \Sigma^{-n} P \rightarrow \Sigma^{-2 n} P$ are 0 and the following diagram commutes:

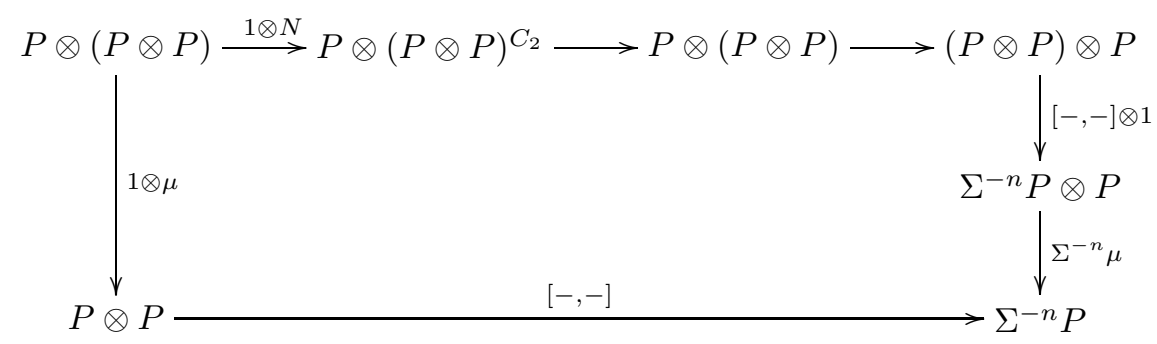

where $N$ is the Norm map of the action by $C_{2}$. The group actions are as before. Clearly, this diagram produces the usual Poisson relation.

Example 8.4. Here we produce examples of functors of type $f_{n}$. As it was mentioned earlier, the real-valued exponential and logarithmic functions are related to the functions $f_{n}$ via the formula $f_{n}(x)=e^{-2^{n-1} \log \left(1-\frac{x}{2^{n-1}}\right)}-1$. The functorial analogue of the right-hand side is, of course, $F_{n}(X):=E\left(\Sigma^{1-n} L\left(\Sigma^{n-1} X\right)\right)$, where $E$ is an exponential functor and $L$ logarithmic. Applying Definitions 7.1 and 4.1 
successively we get a series of isomorphisms:

$$
\begin{aligned}
F_{n}(X \vee Y)=E\left[\Sigma^{1-n} L\left(\Sigma^{n-1}(X \vee Y)\right)\right] \\
\quad \cong E\left[\Sigma^{1-n}\left(L\left(\Sigma^{n-1} X\right) \oplus L\left(\Sigma^{n-1} Y\right) \oplus L\left(U_{L} \Sigma^{n-1} X \otimes U_{L} \Sigma^{n-1} Y\right)\right)\right] \\
\quad \cong E\left[\Sigma^{1-n} L\left(\Sigma^{n-1} X\right)\right] \oplus E\left[\Sigma^{1-n} L\left(\Sigma^{n-1} Y\right)\right] \\
\quad \oplus E\left[\Sigma^{1-n} L\left(U_{L} \Sigma^{n-1} X \otimes U_{L} \Sigma^{n-1} Y\right)\right] \oplus E\left[\Sigma^{1-n} L\left(\Sigma^{n-1} X\right)\right] \otimes E\left[\Sigma^{1-n} L\left(\Sigma^{n-1} Y\right)\right] \\
\quad \oplus E\left[\Sigma^{1-n} L\left(\Sigma^{n-1} X\right)\right] \otimes E\left[\Sigma^{1-n} L\left(U_{L} \Sigma^{n-1} X \otimes U_{L} \Sigma^{n-1} Y\right)\right] \\
\quad \oplus E\left[\Sigma^{1-n} L\left(\Sigma^{n-1} Y\right)\right] \otimes E\left[\Sigma^{1-n} L\left(U_{L} \Sigma^{n-1} X \otimes U_{L} \Sigma^{n-1} Y\right)\right] \\
\quad \oplus E\left[\Sigma^{1-n} L\left(\Sigma^{n-1} X\right)\right] \otimes E\left[\Sigma^{1-n} L\left(\Sigma^{n-1} Y\right)\right] \otimes E\left[\Sigma^{1-n} L\left(U_{L} \Sigma^{n-1} X \otimes U_{L} \Sigma^{n-1} Y\right)\right] \\
\quad \cong F_{n}(X) \oplus F_{n}(Y) \oplus F_{n}\left(\Sigma^{1-n} U_{L} \Sigma^{n-1} X \otimes U_{L} \Sigma^{n-1} Y\right) \oplus F_{n}(X) \otimes F_{n}(Y) \\
\quad \oplus F_{n}(X) \otimes F_{n}\left(\Sigma^{1-n} U_{L} \Sigma^{n-1} X \otimes U_{L} \Sigma^{n-1} Y\right) \\
\quad \oplus F_{n}(Y) \otimes F_{n}\left(\Sigma^{1-n} U_{L} \Sigma^{n-1} X \otimes U_{L} \Sigma^{n-1} Y\right) \\
\quad \oplus F_{n}(X) \otimes F_{n}(Y) \otimes F_{n}\left(\Sigma^{1-n} U_{L} \Sigma^{n-1} X \otimes U_{L} \Sigma^{n-1} Y\right) .
\end{aligned}
$$

Here by $U_{L}$ we denote the functor of type $\frac{x}{1-x}$ associated with the logarithmic functor $L$. In the section on logarithmic functors, this functor is denoted simply by $U$. Here and in what follows we use the subscripts to avoid confusion.

Now we specialize to the case $E=\mathbb{P}$ and $L=\mathbb{L}$. As before, we are omitting the forgetful functors $U_{\log }$ and $U_{e}$ to ease the notation. Thus, everywhere in this paragraph when we write $\mathbb{P} X$ or $\mathbb{L} X$ we mean the underlying modules of the algebras at hand. To see that $F_{n}$ satisfies the defining equation of functors of type $f_{n}$ for this case, we need to show that $U_{F_{n}} X \cong U_{L} \Sigma^{n-1} X$. Observe that $U_{F_{n}} X \cong \mathbb{P}_{1} \Sigma^{n-1} \mathbb{P} \Sigma^{1-n} \mathbb{L} \Sigma^{n-1} X$ and $U_{L} \Sigma^{n-1} X \cong \mathbb{P}_{1} \mathbb{L}\left(\Sigma^{n-1} X\right)$, hence the desired identity follows from the Poincaré-Birkhoff-Witt theorem and the fact that the Lie algebras $\Sigma^{n-1} \mathbb{P} \Sigma^{1-n} \mathbb{L} \Sigma^{n-1} X$ and $\mathbb{L}\left(\Sigma^{n-1} X\right)$ have isomorphic universal enveloping algebras (see also Propositions 3.8 and 3.9 of [12]).

We note that these functors $F_{n}$ take values in the category of $n$-Poisson algebras. An explicit description of the bracket multiplication $[-,-]_{n-1}$ of $F_{n}$ was given in Remark 8.2. As for the Poisson condition, it is evident from the process that we used to extend the map $\Sigma^{1-n} L\left(\Sigma^{n-1} X\right) \otimes \Sigma^{1-n} L\left(\Sigma^{n-1} X\right) \rightarrow \Sigma^{1-n}\left(\Sigma^{1-n} L\left(\Sigma^{n-1} X\right)\right)$ to $\mathbb{P}\left(\Sigma^{1-n} L\left(\Sigma^{n-1} X\right)\right) \otimes \mathbb{P}\left(\Sigma^{1-n} L\left(\Sigma^{n-1} X\right)\right)$.

\section{TAYLOR TOWERS OF FUNCTORS OF TYPE $f_{n}$}

Now we turn to computing the Taylor tower of the functor $F$ of type $f_{n}$. As before, we are going to use induction to compute the layers $D_{k}$. Hence we begin with $D_{2}$. The approach is the same: we start by analyzing the differential $\nabla^{2} F\left(X_{1}, X_{2} ; Y\right)$. Recall that by Proposition 6.1.

$$
\nabla^{2} F\left(X_{1}, X_{2} ; Y\right) \cong D_{1}^{(2)}\left[c r_{3} F(-,-, Y)\right]\left(X_{1}, X_{2}\right) \oplus D_{1}^{(2)}\left[c r_{2} F(-,-)\right]\left(X_{1}, X_{2}\right) .
$$

By the argument employed when discussing the logarithmic functors, we have that

$$
\begin{aligned}
D_{1}^{(2)} & {\left[c r_{3} F(-,-, Y)\right]\left(X_{1}, X_{2}\right) \cong D_{1}^{(2)}\left[c r_{2} F(-\vee-, Y)\right]\left(X_{1}, X_{2}\right) } \\
& \cong D_{1}^{(2)}\left[F(-\vee-) \otimes F(Y) \oplus F\left(\Sigma^{-n+1} U(-\vee-) \otimes U(Y)\right)\right. \\
& \oplus F\left(\Sigma^{-n+1} U(-\vee-) \otimes U(Y)\right) \\
& \otimes(F(-\vee-) \oplus F(Y) \oplus F(-\vee-) \otimes F(Y))]\left(X_{1}, X_{2}\right) .
\end{aligned}
$$


Recall that we are interested in this object only for the case $Y \cong 0$ (see Corollary 6.2). Observe that

$$
D_{1}^{(2)}[F(-\vee-) \otimes F(Y)]\left(X_{1}, X_{2}\right) \cong D_{1}^{(2)}[F(-\vee-)]\left(X_{1}, X_{2}\right) \otimes F(Y) \cong 0
$$

for $Y \cong 0$. The term $D_{1}^{(2)}\left[F\left(\Sigma^{-n+1} U(-\vee-) \otimes U(Y)\right)\right]\left(X_{1}, X_{2}\right)$ also vanishes when evaluated at $Y \cong 0$ by chain rule (Lemma 7.6) and since the functors $F, U, \Sigma$ and $A \otimes-($ for all $A)$ are reduced. Finally, the term

$\left.D_{1}^{(2)} F\left(\Sigma^{-n+1} U(-\vee-) \otimes U(Y)\right) \otimes(F(-\vee-) \oplus F(Y) \oplus F(-\vee-) \otimes F(Y))\right]\left(X_{1}, X_{2}\right)$ is also equivalent to 0 , by chain rule (Lemma 7.6). Thus, for $Y \cong 0$,

$$
D_{1}^{(2)}\left[c r_{3} F(-,-, Y)\right]\left(X_{1}, X_{2}\right) \cong 0 .
$$

Hence it remains to compute $D_{1}^{(2)}\left[c r_{2} F(-,-)\right]\left(X_{1}, X_{2}\right)$.

Observe that each summand of the term

$$
F\left(\Sigma^{-n+1} U X_{1} \otimes U X_{2}\right) \otimes\left[F\left(X_{1}\right) \oplus F\left(X_{2}\right) \oplus F\left(X_{1}\right) \otimes F\left(X_{2}\right)\right]
$$

is 2-reduced in either $X_{1}, X_{2}$ or both. Consequently, $D_{1}^{(2)}$ applied to this term is equivalent to 0 . Hence,

$D_{1}^{(2)}\left[c r_{2} F(-,-)\right]\left(X_{1}, X_{2}\right) \cong D_{1}^{(2)}\left[F(-) \otimes F(-) \oplus F\left(\Sigma^{-n+1} U(-) \otimes U(-)\right)\right]\left(X_{1}, X_{2}\right)$.

First, observe that

$$
D_{1} U(-)(X) \cong D_{1}\left(\mathbb{P}_{1} \Sigma^{n-1} F\right)(-)(X) \cong \Sigma^{n-1} D_{1} F(X) .
$$

The following computations are analogous to those performed for logarithmic functors, so we omit explanations and refer to the logarithmic case for details:

$$
\begin{aligned}
D_{1}^{(2)} & F\left(\Sigma^{-n+1} U\left(X_{1}\right) \otimes U\left(X_{2}\right)\right) \\
& \cong D_{1}^{2}\left[D_{1} F\left(\Sigma^{-n+1} D_{1} U(-)\left(X_{1}\right) \otimes U(-)\right)\right]\left(X_{2}\right) \\
& \cong \Sigma^{-n+1} D_{1} U\left(X_{1}\right) \otimes D_{1}^{2}\left[D_{1} F(\mathbb{K} \otimes U(-))\right]\left(X_{2}\right) \\
& \cong \Sigma^{-n+1} \Sigma^{n-1} D_{1} F\left(X_{1}\right) \otimes D_{1}^{2}\left[D_{1} F(\mathbb{K} \otimes U(-))\right]\left(X_{2}\right) \\
& \cong D_{1} F\left(X_{1}\right) \otimes D_{1} U(-)\left(X_{2}\right) \otimes D_{1} F(\mathbb{K}) \\
& \cong \Sigma^{n-1} D_{1} F\left(X_{1}\right) \otimes D_{1} F\left(X_{2}\right) \otimes D_{1} F(\mathbb{K}) .
\end{aligned}
$$

Using these computations along with Equation (4) of Section 6 which describes the layers of exponential functors (and thus illustrates that $D_{1}^{(2)}$ applied to the summand $F(-) \otimes F(-)$ of $c r_{2} F(-,-)$ equals $\left.D_{1} F(X) \otimes D_{1} F(X)\right)$, we are allowed to conclude that

$$
\nabla^{2} F(X, X ; 0) \cong D_{1} F(X) \otimes D_{1} F(X) \oplus \Sigma^{n-1} D_{1} F(X) \otimes D_{1} F(X) \otimes D_{1} F(\mathbb{K}) .
$$

For the general case of the $k$ 'th layer, observe that by Remark 7.7

$$
\begin{aligned}
& D_{1}^{(k)} c r_{k+1} F(-, \cdots,-, Y)\left(X_{1}, \cdots, X_{k}\right) \\
& \quad \cong D_{1}^{(k)} c r_{k} F(-\vee-,-, \cdots,-, Y)\left(X_{1}, \cdots, X_{k}\right) \\
& \quad \cong \cdots \cong D_{1}^{(k)} c r_{2} F(-\vee \cdots \vee-, Y)\left(X_{1}, \cdots, X_{k}\right) .
\end{aligned}
$$

Hence for $Y \cong 0$, by the above argument for the second differential $\nabla^{2} F$, we have that

$$
D_{1}^{(k)} c r_{2} F(-\vee \cdots \vee-, Y)\left(X_{1}, \cdots, X_{k}\right) \cong 0 .
$$


Consequently, for $Y \cong 0$,

$$
D_{1}^{(k)} c r_{k+1} F(-, \cdots,-, Y)\left(X_{1}, \cdots, X_{k}\right) \cong 0 .
$$

Thus, by Proposition 6.1

$$
\begin{aligned}
\nabla^{k} F\left(X_{1}, \cdots, X_{k} ; 0\right) & \cong D_{1}^{(k)}\left[\operatorname{cr}_{k} F(-, \cdots,-)\right]\left(X_{1}, \cdots, X_{k}\right) \\
& \cong D_{1}^{(k)}\left[c r_{2} F(-\vee \cdots \vee-,-)\right]\left(X_{1}, \cdots, X_{k-1}, X_{k}\right),
\end{aligned}
$$

where the second equivalence is by Remark 7.7 To ease the notation, we define $A_{k}\left(X_{1}, \cdots, X_{k}\right)$ for $k>1$ to be

$$
A_{k}\left(X_{1}, \cdots, X_{k}\right) \stackrel{\text { def }}{=} D_{1}^{(k)}\left[c r_{2} F(-\vee \cdots \vee-,-)\right]\left(X_{1}, \cdots, X_{k-1}, X_{k}\right) .
$$

To compute $A_{k}$, we expand the above second cross effect:

$$
\begin{aligned}
c r_{2} F\left(X_{1} \vee \cdots \vee X_{k-1}, X_{k}\right) \cong & F\left(X_{1} \vee \cdots \vee X_{k-1}\right) \otimes F\left(X_{k}\right) \\
\oplus & F\left(\Sigma^{-n+1} U\left(X_{1} \vee \cdots \vee X_{k-1}\right) \otimes U\left(X_{k}\right)\right) \\
\oplus & F\left(\Sigma^{-n+1} U\left(X_{1} \vee \cdots \vee X_{k-1}\right) \otimes U\left(X_{k}\right)\right) \\
& \otimes\left[F\left(X_{1} \vee \cdots \vee X_{k-1}\right) \oplus F\left(X_{k}\right)\right. \\
& \left.\oplus F\left(X_{1} \vee \cdots \vee X_{k-1}\right) \otimes F\left(X_{k}\right)\right] .
\end{aligned}
$$

We compute $D_{1}^{(k)}$ of the three summands above.

Lemma 9.1. For $k \geq 3$,

$$
D_{1}^{(k)} F\left(X_{1} \vee \cdots \vee X_{k-1}\right) \otimes F\left(X_{k}\right) \cong A_{k-1}\left(X_{1}, \cdots, X_{k-1}\right) \otimes D_{1} F\left(X_{k}\right),
$$

with $A_{2} \cong D_{1} F\left(X_{1}\right) \otimes D_{1} F\left(X_{2}\right) \oplus \Sigma^{n-1} D_{1} F\left(X_{1}\right) \otimes D_{1} F\left(X_{2}\right) \otimes D_{1} F(\mathbb{K})$, as computed above.

Proof. The proof of this lemma follows from the following straightforward sequence of identities:

$$
\begin{aligned}
D_{1}^{(k)} & F\left(X_{1} \vee \cdots \vee X_{k-1}\right) \otimes F\left(X_{k}\right) \\
& \cong D_{1}^{(k-1)} F(-\vee \cdots \vee-)\left(X_{1}, \cdots, X_{k-1}\right) \otimes D_{1} F\left(X_{k}\right) \\
& \cong D_{1}^{(k-1)} c r_{2} F\left(X_{1} \vee \cdots \vee X_{k-2}, X_{k-1}\right) \otimes D_{1} F\left(X_{k}\right) \\
& \cong A_{k-1}\left(X_{1}, \cdots, X_{k-1}\right) \otimes D_{1} F\left(X_{k}\right) .
\end{aligned}
$$

Lemma 9.2. For all $k>2$,

$$
\begin{aligned}
& D_{1}^{(k)} F\left(\Sigma^{-n+1} U(-\vee \cdots \vee-) \otimes U(-)\right)\left(X_{1}, \cdots, X_{k}\right) \\
\cong & \bigoplus_{\sigma \in \Sigma_{k-1}} D_{1} F\left(X_{\sigma(1)}\right) \otimes \cdots \otimes D_{1} F\left(X_{\sigma(k-1)}\right) \otimes D_{1} F\left(X_{k}\right) \otimes \Sigma^{(k-1)(n-1)} D_{1} F(\mathbb{K}) .
\end{aligned}
$$

Proof. Since it does not matter in which order we apply $D_{1}$ 's, we begin by computing $D_{1}^{k}$, i.e. the derivative with respect to the variable $X_{k}$ :

$$
\begin{array}{rl}
D_{1}^{k} & F\left(\Sigma^{-n+1} U\left(X_{1} \vee \cdots \vee X_{k-1}\right) \otimes U(-)\right)\left(X_{k}\right) \\
& \cong D_{1} F\left(\Sigma^{-n+1} U\left(X_{1} \vee \cdots \vee X_{k-1}\right) \otimes D_{1} U(-)\left(X_{k}\right)\right) \\
& \cong \Sigma^{-n+1} U\left(X_{1} \vee \cdots \vee X_{k-1}\right) \otimes \Sigma^{n-1} D_{1} F\left(X_{k}\right) \otimes D_{1} F(\mathbb{K}) \\
& \cong U\left(X_{1} \vee \cdots \vee X_{k-1}\right) \otimes D_{1} F\left(X_{k}\right) \otimes D_{1} F(\mathbb{K}) .
\end{array}
$$


Hence, recalling that $U$ is a functor of type $\frac{x}{1-x}$ and using our computations for these functors, we get

$$
\begin{aligned}
D_{1}^{(k)} & F\left(\Sigma^{-n+1} U\left(X_{1} \vee \cdots \vee X_{k-1}\right) \otimes U\left(X_{k}\right)\right) \\
& \cong D_{1}^{(k-1)} U\left(X_{1} \vee \cdots \vee X_{k-1}\right) \otimes D_{1} F\left(X_{k}\right) \otimes D_{1} F(\mathbb{K}) \\
& \cong \bigoplus_{\sigma \in \Sigma_{k-1}} D_{1} U\left(X_{\sigma(1)}\right) \otimes \cdots \otimes U\left(X_{\sigma(k-1)}\right) \otimes D_{1} F\left(X_{k}\right) \otimes D_{1} F(\mathbb{K}) \\
& \cong \bigoplus_{\sigma \in \Sigma_{k-1}} D_{1} F\left(X_{\sigma(1)}\right) \otimes \cdots \otimes F\left(X_{\sigma(k-1)}\right) \otimes D_{1} F\left(X_{k}\right) \otimes \Sigma^{(k-1)(n-1)} D_{1} F(\mathbb{K}) .
\end{aligned}
$$

The final piece in which we are interested is

$$
\begin{aligned}
& D_{1}^{(k)} F\left(\Sigma^{-n+1} U\left(X_{1} \vee \cdots \vee X_{k-1}\right) \otimes U\left(X_{k}\right)\right) \\
& \quad \otimes\left[F\left(X_{1} \vee \cdots \vee X_{k-1}\right) \oplus F\left(X_{k}\right) \oplus F\left(X_{1} \vee \cdots \vee X_{k-1}\right) \otimes F\left(X_{k}\right) .\right.
\end{aligned}
$$

In other words we need to compute $D_{1}^{(k)}$ of the three summands above. Observe that the second and and the third summands are at least 2-reduced in the variable $X_{k}$. Hence, $D_{1}^{k}$ applied to these summands produces objects equivalent to 0 . Consequently, it remains to compute only

$$
D_{1}^{(k)}\left[F\left(\Sigma^{-n+1} U\left(X_{1} \vee \cdots \vee X_{k-1}\right) \otimes U\left(X_{k}\right)\right) \otimes F\left(X_{1} \vee \cdots \vee X_{k-1}\right)\right] .
$$

We begin by applying $D_{1}^{k}$ to get

$$
\begin{aligned}
& \Sigma^{-n+1} U\left(X_{1} \vee \cdots \vee X_{k-1}\right) \otimes D_{1} U\left(X_{k}\right) \otimes D_{1} F(\mathbb{K}) \otimes F\left(X_{1} \vee \cdots \vee X_{k-1}\right) \\
& \quad \cong U\left(X_{1} \vee \cdots \vee X_{k-1}\right) \otimes F\left(X_{1} \vee \cdots \vee X_{k-1}\right) \otimes D_{1} F\left(X_{k}\right) \otimes D_{1} F(\mathbb{K}) .
\end{aligned}
$$

Thus, we need to understand

$$
D_{1}^{(k-1)}\left[U\left(X_{1} \vee \cdots \vee X_{k-1}\right) \otimes F\left(X_{1} \vee \cdots \vee X_{k-1}\right)\right] .
$$

Recall that $U$ is a functor of type $\frac{x}{1-x}$ and hence $U\left(X_{1} \vee \cdots \vee X_{k-1}\right)$ can be expanded as a sum. Note that the only terms of $U\left(X_{1} \vee \cdots \vee X_{k-1}\right)$ which are not at least 2 -reduced in at least one of the variables are

$U\left(X_{1}\right) \oplus \cdots \oplus U\left(X_{k-1}\right) \oplus \bigoplus_{i \neq j} U\left(X_{i}\right) \otimes U\left(X_{j}\right) \oplus \cdots \oplus \bigoplus_{\sigma \in \Sigma_{k-1}} U\left(X_{\sigma(1)}\right) \otimes U\left(X_{\sigma(k-1)}\right)$.

A typical summand in the above sum is of the form

$$
U\left(X_{i_{1}}\right) \otimes \cdots \otimes U\left(X_{i_{s}}\right),
$$

with $s \leq k-1$ and $i_{l} \neq i_{t}$ for $l \neq t$. Consequently, it is enough to understand

$$
D_{1}^{(k-1)}\left[U\left(X_{i_{1}}\right) \otimes \cdots \otimes U\left(X_{i_{s}}\right) \otimes F\left(X_{1} \vee \cdots \vee X_{k-1}\right)\right] .
$$

To simplify the indexing, let us assume that in the above term $i_{j}=j$. In other words, we compute

$$
\begin{aligned}
D_{1}^{(k-1)} & {\left[U\left(X_{1}\right) \otimes \cdots \otimes U\left(X_{s}\right) \otimes F\left(X_{1} \vee \cdots \vee X_{k-1}\right)\right] } \\
& \cong D_{1}^{(k-1)}\left[U ( X _ { 1 } ) \otimes \cdots \otimes U ( X _ { s } ) \otimes \left(F\left(X_{1}\right) \oplus F\left(X_{2} \vee \cdots \vee X_{k-1}\right)\right.\right. \\
& \oplus F\left(\Sigma^{-n+1} U\left(X_{1}\right) \otimes F\left(X_{2} \vee \cdots \vee X_{k-1}\right)\right. \\
& \left.\left.\oplus F\left(\Sigma^{-n+1} U\left(X_{1}\right) \otimes F\left(X_{2} \vee \cdots \vee X_{k-1}\right)\right) \otimes(\cdots)\right)\right] \\
& \cong D_{1}^{(k-1)}\left[U\left(X_{1}\right) \otimes \cdots \otimes U\left(X_{s}\right) \otimes F\left(X_{2} \vee \cdots \vee X_{k-1}\right)\right]
\end{aligned}
$$


since all the other terms are at least 2-reduced in $X_{1}$. Iterating this process for $X_{2}, \cdots, X_{s}$, we get that

$$
\begin{aligned}
& D_{1}^{(k-1)}\left[U\left(X_{1}\right) \otimes \cdots \otimes U\left(X_{s}\right) \otimes F\left(X_{1} \vee \cdots \vee X_{k-1}\right)\right] \\
& \quad \cong D_{1}^{(k-1)}\left[U\left(X_{1}\right) \otimes \cdots \otimes U\left(X_{s}\right) \otimes F\left(X_{s+1} \vee \cdots \vee X_{k-1}\right)\right] \\
& \quad \cong D_{1} U\left(X_{1}\right) \otimes \cdots \otimes D_{1} U\left(X_{s}\right) \otimes A_{k-s-1}\left(X_{s+1}, \cdots, X_{k-1}\right) \\
& \quad \cong \Sigma^{s(n-1)} D_{1} F\left(X_{1}\right) \otimes \cdots \otimes D_{1} F\left(X_{s}\right) \otimes A_{k-s-1}\left(X_{s+1}, \cdots, X_{k-1}\right),
\end{aligned}
$$

where $A_{k-s-1}$ is as it is defined in Equation (11).

We combine these computations in the following lemma by first agreeing to augment Equation (11) by defining $A_{1}(X) \stackrel{\text { def }}{=} D_{1} F(X)$.

Lemma 9.3. For all $k>2$ and $X_{1}=\cdots=X_{k}=X$, and by abbreviating $A_{k-s-1}(X, \cdots, X)$ by $A_{k-s-1}$, we have

$$
\begin{aligned}
D_{1}^{(k)} & F\left(\Sigma^{-n+1} U(X \vee \cdots \vee X) \otimes U(X)\right) \\
& \otimes[F(X \vee \cdots \vee X) \oplus F(X) \oplus F(X \vee \cdots \vee X) \otimes F(X)] \\
& \cong \bigoplus_{s=1}^{k-2} \Sigma^{s(n-1)}\left[\bigoplus_{\sigma \in P(k-1, s)} \bigotimes_{1}^{s} D_{1} F(X) \otimes A_{k-s-1}\right] \otimes D_{1} F(X) \otimes D_{1} F(\mathbb{K}),
\end{aligned}
$$

where $P(k-1, s)$ is the set of $s$ different ordered letters from a collection of $k-1$ letters.

The above Lemmas $9.1,9.2$, and 9.3 give a complete description of the differentials $\nabla^{k} F(X, \cdots, X ; 0)$, which are of course the essential ingredients for computing the layers by Corollary 6.2. We agree to augment Equation (11) further by setting $A_{0}=\mathbb{K}$ and combining these lemmas to obtain the following proposition.

Proposition 9.4. The differentials of functors of type $f_{n}$ are as follows.

For $k=2$,

$$
\begin{aligned}
& \nabla^{2} F(X, X ; 0) \cong A_{2} \\
& \quad \cong D_{1} F(X) \otimes D_{1} F(X) \oplus \Sigma^{n-1} D_{1} F(X) \otimes D_{1} F(X) \otimes D_{1} F(\mathbb{K}) .
\end{aligned}
$$

For $k>2$,

$$
\begin{aligned}
& \nabla^{k} F(X, \cdots, X ; 0) \cong A_{k} \\
& \quad \cong A_{k-1} \otimes D_{1} F(X) \\
& \quad \oplus \bigoplus_{s=1}^{k-1} \Sigma^{s(n-1)}\left[\bigoplus_{\sigma \in P(k-1, s)}\left[\bigotimes_{1}^{s} D_{1} F(X)\right] \otimes A_{k-s-1}\right] \otimes D_{1} F(X) \otimes D_{1} F(\mathbb{K}),
\end{aligned}
$$

where, as before, $P(k-1, s)$ is the set of $s$ different ordered letters from a collection of $k-1$ letters, while $A_{0}=\mathbb{K}$ and $A_{1}=D_{1} F(X)$.

\section{Functional EQuations And operads}

We have already commented on the connection between functors of type $f_{n}$ and the homology of little $n$-cubes operads, which was suggested by the fact that they have the same set of associated algebras. In fact, it is in part with this relationship in mind that we considered these functors. The following discussion is intended to explore this relationship further. 
As an easy consequence of the defining equation of functors of type $f_{n}$ (Definition 8.1), we note that with an additional hypothesis that $F$ preserves connectivity (this happens for example when $F$ is a continuous functor, e.g. see [8]) as $n$ increases, so does the connectivity of the term

$$
F\left(\Sigma^{-n+1} U X \otimes U Y\right) \cong F\left(\Sigma^{-n+1} \mathbb{P}_{1} \Sigma^{n-1} F(X) \otimes \mathbb{P}_{1} \Sigma^{n-1} F(Y)\right) .
$$

Thus, the equation degenerates to the defining equation of exponential functors. This, of course, is in agreement with the fact that as a function of real variables $e^{x}-1$ is the limit of the sequence of functions $\left\{f_{n}\right\}$. Moreover, by analyzing the differentials $D_{n}$ of functors of type $f_{n}$ that we just computed, it is evident that the connectivity of the terms

$$
\bigoplus_{s=1}^{k-1} \Sigma^{s(n-1)}\left[\bigoplus_{\sigma \in P(k-1, s)}\left[\bigotimes_{1}^{s} D_{1} F(X)\right] \otimes A_{k-s-1}\right] \otimes D_{1} F(X) \otimes D_{1} F(\mathbb{K})
$$

of $D_{n}$ increases with $n$, and hence in the limit case the layers are the same as the layers of exponential functors.

However, if we set $n=1$ in the equation of Definition 8.1 (we will call such functors functors of type $f_{1}$ ), we get functors that factor through the category of usual Poisson algebras, while the functors of type $\frac{x}{1-x}$ (Definition 5.1) factor through the category of associative algebras, and of course, the associative and Poisson algebras are quite different. A similar situation occurs in [5] where E. Getzler and J.D.S. Jones prove that the homology little $n$-cubes operads $\mathbf{e}_{\mathbf{n}}$ are isomorphic to the Poisson operads $\mathbf{p}_{\mathbf{n}}$ for $n>1$ (Theorem 1.6 of [5]), while $\mathbf{e}_{\mathbf{1}}$ is not isomorphic to $\mathbf{p}_{\mathbf{1}}$. However, $\mathbf{e}_{\mathbf{1}}$ is naturally a filtered operad, and the associated graded operad is the Poisson operad $\mathbf{p}_{\mathbf{1}}$ (Section 5.3 of $[5]$ ). We have a similar result. To make this statement precise, let us consider the functors into $C h(\mathbb{K})$ of type $\frac{x}{1-x}$ again. We already showed that if $F$ is such a functor, then $F(X)$ is naturally an associative algebra. In fact, it is equipped with an even richer algebraic structure if we restrict ourselves to functors whose domain is $C h(\mathbb{K})$. Using the fact that finite coproducts are also products in $C h(\mathbb{K})$, we define a comultiplication map $\Delta_{F(X)}: F(X) \rightarrow F(X) \otimes F(X)$ for such functors:

$$
F(X) \stackrel{\Delta}{\rightarrow} F(X \vee X) \rightarrow F(X) \otimes F(X) \oplus F(X) \otimes F(X) \stackrel{+}{\rightarrow} F(X) \otimes F(X) .
$$

Combined with the associative multiplication defined earlier, $\Delta_{F(X)}$ makes $F(X)$ into a Hopf algebra. In addition, from the symmetry of the definition of $\Delta_{F(X)}$ it is immediate that $F(X)$ is cocommutative. Thus, $F$ factors through the category $\mathcal{C} H_{\mathbb{K}}$ of cocommutative Hopf algebras. Hence, by the structure theorem of Milnor and Moor [12], $F(X)$ is isomorphic to the universal enveloping algebra $U(L)$ of some Lie algebra $L$. In fact, this can be done functorially to produce a commutative diagram

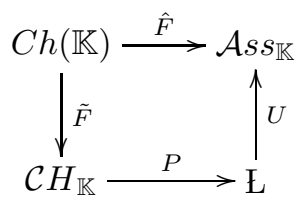

where $\hat{F}$ exists by Lemma $5.3, \tilde{F}$ by the argument above, and $P$ is the inverse functor of the universal enveloping algebra functor $U$ considered as a functor into 
$\mathcal{C} H_{\mathbb{K}}$ (Theorem 5.18 of [12]). Here, as before, $\mathcal{L}_{\mathbb{K}}$ is the category of Lie algebras over the field $\mathbb{K}$ of characteristic 0 .

Now we define a filtration on $F(X)$ which is a essentially the Lie filtration of [12] (see Definition 5.12 of [12]). In other words, roughly speaking, it is the filtration of $F(X)$ given by powers of $P \circ \tilde{F}(X)$, thought of as a subobject of $\hat{F}(X)$ via the universal enveloping algebra functor, i.e. $F_{p}(X)=\sum_{q \leq p}[P \circ \tilde{F}(X)]^{q}$. More precisely,

$$
\begin{gathered}
F_{1}(X)=\operatorname{Im}[\mathbb{K} \oplus P \circ \tilde{F}(X) \rightarrow \hat{F}(X)], \\
F_{p+1}(X)=\operatorname{Im}\left[F_{p}(X) \oplus\left((P \circ \tilde{F}(X)) \otimes F_{p}(X)\right) \rightarrow \hat{F}(X)\right] .
\end{gathered}
$$

The associated graded object is defined to be $A(X)=F_{1} \oplus F_{2} / F_{1} \oplus \cdots F_{p} / F_{p-1} \oplus \cdots$. Now we state the promised result.

Proposition 10.1. If $F: C h(\mathbb{K}) \rightarrow C h(\mathbb{K})$ is a functor of type $\frac{x}{1-x}$, then the correspondence $X \longmapsto A(X)$ defines a functor of type $f_{1}$, i.e. it defines a functor that satisfies the conditions of Definition 8.1 for the case $n=1$.

Proof. Throughout this proof we omit the forgetful functors where no confusion may arise to ease the notation. Thus, for example, when we refer to a functor $F$ into Lie algebras as a logarithmic functor, naturally we mean $F$ composed with the forgetful functor $U_{l o g}$ from Lie algebras to chain complexes, as by definition, all our functors of different types have the category $C h(\mathbb{K})$ as their target.

First observe that $A(X)$ is a functor, i.e. that any map $f: X \rightarrow Y$ in $C h(\mathbb{K})$ induces a morphism of filtered algebras. Indeed, as $\tilde{F}$ and $P$ are functors, we have a map $\mathbb{T}_{1} \circ P \circ \tilde{F}(X) \rightarrow \mathbb{T}_{1} \circ P \circ \tilde{F}(Y)$, which takes components of degree $\leq q$ to components of degree $\leq q$. Recall that the universal enveloping functor $U$ on a Lie algebra $\mathrm{g}$ can be constructed as a quotient of $\mathbb{T}_{1} \mathrm{~g}$ by the ideal generated by relations $i([x, y])=i(x) i(y)-i(y) i(x)$, where $i: \mathbf{g} \rightarrow \mathbb{T}_{1} \mathbf{g}$ is the obvious embedding, and $x, y \in \mathrm{g}$. Hence we have that $f^{*}\left(F_{p}(X)\right) \subseteq F_{p}(Y)$, where $f^{*}$ is the map induced by $f$. Thus, $f$ produces a morphism of filtered algebras which in its turn induces a map of corresponding graded objects $A(X) \rightarrow A(Y)$ making $A$ into a functor.

It is a well-known fact about Lie algebras that the graded algebra associated to the Lie filtration (that we just presented) is graded commutative (see Section 7.3 of [16 for example). Moreover, if we denote by $S$ the functor that takes the module underlying the Lie algebra $\mathbf{g}$ to the chain complex underlying the graded algebra associated with the Lie filtration of $U(\mathbf{g})$, then it satisfies the equation $S(\mathbf{g} \oplus \mathbf{h}) \cong$ $S(\mathbf{g}) \oplus S(\mathbf{h}) \oplus S(\mathbf{g}) \otimes S(\mathbf{h})$, where $\mathbf{g} \oplus \mathbf{h}$ is the coproduct of $\mathbf{g}$ and $\mathbf{h}$ in $C h(\mathbb{K})$, with the Lie bracket defined in the obvious way: $[(a, b),(\bar{a}, \bar{b})]=\left([a, \bar{a}]_{\mathbf{g}},[b, \bar{b}]_{\mathbf{h}}\right)$. This is the case since in characteristic 0 , the graded commutative algebra associated to the Lie filtration of $U(\mathbf{g})$ is isomorphic to the free symmetric algebra on $\mathbf{g}$. Thus, $S$ is an exponential functor.

To complete the proof of the proposition we simply observe that the functor $A$ is the composition of $S$ with $P \circ \tilde{F}$, and since $P \circ \tilde{F}$ is a coproduct preserving functor into Lie algebras ( $P$ is coproduct preserving by Theorem 5.9 of [12]), and thus logarithmic (see Remark 7.4), the computations done in Example8.4 for $n=1$ imply that $A=S \circ P \circ \tilde{F}$ satisfies the defining equation of functors of type $f_{1}$, i.e the defining equation of Definition 8.1 for the case $n=1$.

We conclude by considering the example of the forgetful functor from the category of $n$-Poisson algebras in greater detail. Its relevance to the algebras over 
the homology of little $n$-cubes operads, as well as to the homology of configuration spaces, is the main reason for our interest.

Recall that the triple associated with the category of $n$-Poisson algebras is of the form

$$
T_{n}(X)=\bigoplus_{k} \mathbf{p}_{\mathbf{n}}(k) \otimes_{\Sigma_{k}} X^{\otimes k}
$$

where $\mathbf{p}_{\mathbf{n}}$ is the $n$-Poisson operad. The algebras over this triple are precisely the $n$-Poisson algebras. Of course, for $n \geq 2$ the operads $\mathbf{p}_{\mathbf{n}}$ are equivalent to the homology of little $n$-cubes operads $\mathbf{e}_{\mathbf{n}}$ (e.g. see [5]). Note that we can read the operad off the triple (12) by computing the differentials evaluated at the ground field $\mathbb{K}$ viewed as a chain complex concentrated in degree 0 :

$$
\nabla^{k} F(\mathbb{K}, \cdots, \mathbb{K} ; 0)
$$

Recalling that by Example 8.4 functors $T_{n}$ are of type $f_{n}$, and observing that $D_{1} T_{n}(\mathbb{K}) \cong \mathbb{K}$, we can rewrite the Proposition 9.4 as follows:

For $k=2$,

$$
\nabla^{2} T_{n}(\mathbb{K}, \mathbb{K} ; 0) \cong A_{2} \cong \mathbb{K} \oplus \Sigma^{n-1} \mathbb{K}
$$

For $k>2$,

$$
\nabla^{k} T_{n}(\mathbb{K}, \cdots, \mathbb{K} ; 0) \cong A_{k} \cong A_{k-1} \oplus \bigoplus_{s=1}^{k-1} \Sigma^{s(n-1)}\left[\bigoplus_{\sigma \in P(k-1, s)} A_{k-s-1}\right]
$$

where $A_{i}$ and $P(k-1, s)$ are as before. In fact, we can provide a better description.

Lemma 10.2. For $k \geq 2$,

$$
\nabla^{k} T_{n}(\mathbb{K}, \cdots, \mathbb{K} ; 0) \cong \bigotimes_{j=1}^{k-1} H^{*}\left(\bigvee_{j} S^{n-1}\right)
$$

where on the right-hand side we have the tensor product of cohomologies of the wedge of $j$ copies of the sphere $S^{n-1}$. Here and in what follows the homologies are taken with coefficients in the field $\mathbb{K}$.

Proof. We prove by induction on $k$. Since we intend to use the formulas (13) and (14), we have to consider both $k=2$ and $k=3$ for the base case.

For $k=2$, we need to show that $\nabla^{2} T_{n}(\mathbb{K}, \mathbb{K} ; 0) \cong H^{*}\left(S^{n-1}\right)$. This is immediate from Equation (13).

For $k=3$, note that $\bigotimes_{j=1}^{2} H^{*}\left(\bigvee_{j} S^{n-1}\right) \cong H^{*}\left(S^{n-1}\right) \otimes H^{*}\left(S^{n-1} \vee S^{n-1}\right)$. Hence by Mayer-Vietoris (e.g. see [1]), the right-hand side of Equation (15) has a copy of $\mathbb{K}$ in degree 0 , three copies of $\mathbb{K}$ in degree $n-1$ and two copies of $\mathbb{K}$ in degree $2(n-1)$. On the other hand by Equations (13) and (14),

$$
\nabla^{3} T_{n}(\mathbb{K}, \mathbb{K}, \mathbb{K} ; 0) \cong \mathbb{K} \oplus \Sigma^{n-1} \mathbb{K} \oplus \Sigma^{n-1}(\mathbb{K} \oplus \mathbb{K}) \oplus \Sigma^{2(n-1)}(\mathbb{K} \oplus \mathbb{K}),
$$

which proves the case $k=3$. 
Suppose the lemma holds for $k=l-1$. For $k=l$, by Mayer-Vietoris, on the right-hand side of Equation (15) we have

$$
\begin{aligned}
\bigotimes_{j=1}^{l-1} & H^{*}\left(\bigvee_{j} S^{n-1}\right) \\
& \cong \bigotimes_{j=1}^{l-2} H^{*}\left(\bigvee_{j} S^{n-1}\right) \otimes H^{*}\left(\bigvee_{l-1} S^{n-1}\right) \cong \bigotimes_{j=1}^{l-2} H^{*}\left(\bigvee_{j} S^{n-1}\right) \otimes\left[\mathbb{K} \oplus \Sigma^{n-1} \mathbb{K}^{\oplus l-1}\right] \\
& \cong A_{l-1} \otimes\left[\mathbb{K} \oplus \Sigma^{n-1} \mathbb{K}^{\oplus l-1}\right] \cong A_{l-1} \otimes \mathbb{K} \oplus A_{l-1} \otimes \Sigma^{n-1} \mathbb{K}^{\oplus l-1} \\
& \cong A_{l-1} \oplus\left[A_{l-2} \oplus \bigoplus_{s=1}^{l-2} \Sigma^{s(n-1)}\left(\bigoplus_{P(l-2, s)} A_{l-s-2}\right)\right] \otimes \Sigma^{n-1} \mathbb{K}^{\oplus l-1} \\
& \cong A_{l-1} \oplus A_{l-2} \otimes \Sigma^{n-1} \mathbb{K}^{\oplus l-1} \oplus \bigoplus_{s=1}^{l-2} \Sigma^{(s+1)(n-1)}\left[\bigoplus_{P(l-2, s)} A_{l-s-2} \otimes \mathbb{K}^{\oplus l-1}\right]
\end{aligned}
$$

We renumber the sum in the last equation by setting $s+1=t$ to get

$$
\begin{aligned}
& \bigotimes_{j=1}^{l-1} H^{*}\left(\bigvee_{j} S^{n-1}\right) \\
& \quad \cong A_{l-1} \oplus A_{l-2} \otimes \Sigma^{n-1} \mathbb{K}^{\oplus l-1} \oplus \bigoplus_{t=2}^{l-1} \Sigma^{t(n-1)}\left[\bigoplus_{P(l-2, t-1)} A_{l-t-1} \otimes \mathbb{K}^{\oplus l-1}\right] .
\end{aligned}
$$

We abuse the notation to denote by $P(l-2, t-1)$ the number of elements in $P(l-2, t-1)$. Then

$$
P(l-2, t-1)(l-1)=\frac{(l-2) !}{(l-t-1) !}(l-1)=\frac{(l-1) !}{(l-t-1) !}=P(l-1, t) .
$$

These identities allow us to simplify our equations further:

$$
\begin{aligned}
& \bigotimes_{j=1}^{l-1} H^{*}\left(\bigvee_{j} S^{n-1}\right) \\
& \cong A_{l-1} \oplus A_{l-2} \otimes \Sigma^{n-1} \mathbb{K}^{\oplus l-1} \oplus \bigoplus_{t=2}^{l-1} \Sigma^{t(n-1)}\left[\bigoplus_{P(l-1, t)} A_{l-t-1}\right] \\
& \cong A_{l-1} \oplus \bigoplus_{t=1}^{l-1} \Sigma^{t(n-1)}\left[\bigoplus_{P(l-1, t)} A_{l-t-1}\right] \cong A_{l} \cong \nabla^{l} T_{n}(\mathbb{K}, \cdots, \mathbb{K} ; 0),
\end{aligned}
$$

thus completing the proof of the lemma.

Recall that one of the descriptions of the homology of little $n$-cubes operads is via configuration spaces (see Section 2), which is the reason why our Lemma 10.2 is reminiscent of results of F. Cohen (see [2]), who applied a theorem by Fadell and Neuwirth (see [4]) to describe the homology of configuration spaces of embedding into $\mathbb{R}^{n}$. 
Lemma 10.3 (Cohen). Additively,

$$
H^{*}\left(F_{n}(k)\right) \cong \bigotimes_{j=1}^{k-1} H^{*}\left(\bigvee_{j} S^{n-1}\right),
$$

where $F_{n}(k)$ is the configuration space of embedding into $\mathbb{R}^{n}$ as it is defined in Section 2

Consequently, these computations of homology of configuration spaces can be recovered from Lemma 10.2 Similarly, our techniques can be used to reproduce the description of the action of symmetric groups on $H^{*}\left(F_{n}(k)\right)$ (Section 7 of [2]).

\section{ACKNOWLEDGMENTS}

The author thanks Randy McCarthy for his encouragement and many helpful suggestions, and the anonymous referee whose valuable comments greatly improved this paper.

\section{REFERENCES}

[1] G.Bredon. Topology and Geometry. New York: Springer-Verlag, 1993 MR 1224675 (94d:55001)

[2] F.Cohen. The Homology of $\mathcal{C}_{n+1}$-spaces, $n \geq 0$ in "The homology of iterated loop spaces". Lecture Notes in Math., 533, 207-351. 1976 MR0436146 (55:9096)

[3] A.D.Elmendorf, I.Kriz, M.A.Mandell and J.P.May. Rings, Modules, and Algebras in Stable Homotopy Theory. Mathematical Surveys and Monographs. Vol. 47. AMS. 1996 MR 1417719 (97h:55006)

[4] E.Fadell and L.Neuwirth. Configuration Spaces. Math. Scan., 10, 119-126. 1962 MR0141126 $(25: 4537)$

[5] E.Getzler and J.D.S.Jones. Operads, Homotopy Algebra, and Iterated Integrals for Double Loop Spaces. Preprint. 1993

[6] T.Goodwillie. Calculus I: The First Derivative of Pseudoisotopy Theory. K-theory, 4, 1-27. 1990 MR1076523 (92m:57027)

[7] T.Goodwillie. Calculus II: Analytic Functors. K-theory, 5, 295-332. 1992 MR 1162445 (93i:55015)

[8] T.Goodwillie. Calculus III: The Taylor Series of a Homotopy functor. Geometry and Topology, 7, 645-711. 2003 MR2026544 (2005e:55015)

[9] B.Johnson and R.McCarthy. Deriving Calculus with Cotriples. Trans. Amer. Math. Soc., 356, 757-804. 2004 MR 2022719

[10] I.Kriz and J.P.May. Operads, Algebras, Modules and Motives. Astérisque, 233, 1995 MR $1361938(96 \mathrm{j}: 18006)$

[11] R.McCarthy and V.Minasian. On Triples, Operads and Generalized Homogeneous Functors. Preprint, 2004

[12] J.Milnor and J.C.Moor. On the Structure of Hopf Algebras. Ann. of Math. (2), 81, 211-264. 1965 MR0174052 (30:4259)

[13] V.Minasian. André-Quillen spectral sequence for THH. Topology Appl., 129, 273-280. 2003 MR 1962984(2004c:55018)

[14] D.G.Quillen. On the (co-)homology of commutative rings. AMS Proc Symp. Pure Math., 17, 65-87. 1970 MR0257068 (41:1722)

[15] A.Robinson. Gamma homology, Lie representations and $E_{\infty}$ multiplications. Invent. Math., 152, 331-348. 2003 MR 1974890|(2004c:55020)

[16] C.A.Weibel. An Introduction to Homological Algebra. Cambridge Studies in Advanced Mathematics, 38. 1994 MR1269324 (95f:18001)

Department of Mathematics, Brown University, Providence, Rhode Island 02912-1917

E-mail address: minasian@math.brown.edu 